\title{
Isolation and Structural Characterization of Regioisomers of Di- brominated Terrylene Diimides
}

\author{
Shivangee Jha, Kundan Singh Mehra, Avantika Hasija, Deepak Chopra, Ramprasad Regar and \\ Jeyaraman Sankar* \\ Department of Chemistry, Indian Institute of Science Education and Research (IISER) Bhopal, Bhopal Bypass \\ Road, Bhopal - 462066, India \\ Correspondence to sankar@iiserb.ac.in
}

\section{CONTENTS}

1. Materials and Methods

2. Synthetic Procedures and Characterization Data

3. NBO calculation data

$\mathrm{S} 4$

4. NMR Spectra

S5-S15

5. HRMS Spectra

S5-S18

6. Photo-physical studies

S18-S19

7. Electrochemical studies

S19-S21

8. X-ray diffraction structures

S22 


\section{1: Materials and Methods:}

All the reactions were carried out in oven-dried glassware under argon atmosphere wherever required. All the chemicals received from Sigma Aldrich and Spectrochem. All solvents were dried according to standard methods prior to use. For all the optical and electrochemical measurements spectroscopic grade solvents were used. An oil bath was used wherever elevation of reaction temperature was needed. Compounds synthesized were purified by column chromatography followed by recrystallization. ${ }^{1} \mathrm{H}$ and ${ }^{13} \mathrm{C}$ NMR spectra were recorded on Bruker Avance $500 \mathrm{MHz}$ and $700 \mathrm{MHz}$ instruments using $\mathrm{CDCl}_{3}$ and Benzene- $\mathrm{d}_{6}$ as solvents. Chemical shifts were given in parts per million (ppm), relative to the residual solvent signals. Abbreviations were used to indicate multiplicity: $s$ (singlet), $d$ (doublet), $t$ (triplet), dd (doublet of doublet), td (triplet of doublet) and m (multiplet). High resolution mass spectrometry (HRMS) atmospheric pressure chemical ionization (APCI) mass spectra were recorded on a Bruker micro TOF-QII mass spectrometer. Steady-state absorption recorded on Perkin Elmer LAMBDA 950 UV/VIS/NIR spectrophotometer with $10 \mathrm{~mm}$ path length quartz cuvette, and fluorescence emission recorded on Horiba Fluorolog spectrophotometer. Cyclic voltammetry (CV) experiment performed on $\mathrm{CHI}$ instrument using $\mathrm{CH}$ potentiostat. $\mathrm{CV}$ experiments done using conventional three-electrode system consists of a Pt-disk as working electrode, saturated calomel electrode (SCE) as reference electrode, and Pt-wire as counter electrode and all the data recorded in dry chloroform solution using $0.1 \mathrm{M}$ Tetrabutylammonium hexafluorophosphate (TBAPF) as supporting electrolyte. Single crystal X-ray diffraction (SCXRD) measurements were carried out at $100 \mathrm{~K}$ on Bruker Apex diffractometer with a CCD detector with Mo-K $\alpha$ radiation. Density functional theory (DFT) calculations were performed using the Gaussian 09 program with the B3LYP exchange-correlation functional. Charge distribution of the molecules was calculated from NBO (Natural Bond Orbital).[1]

\section{2: Synthetic Procedures and characterization data:}

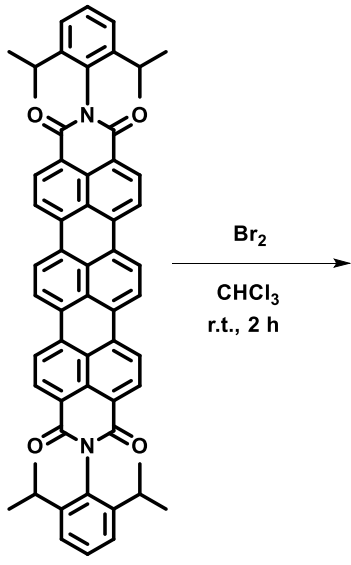

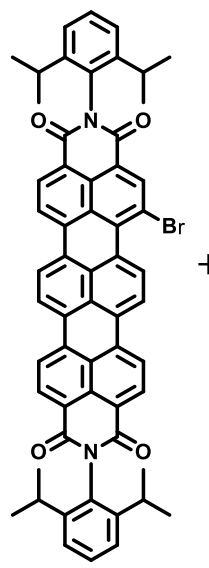

1-Br

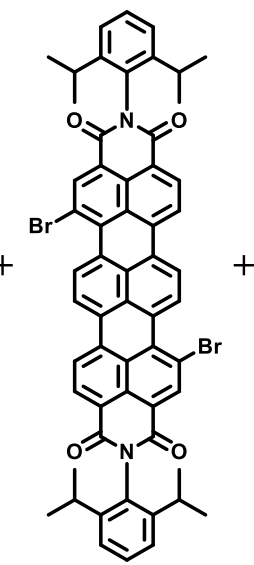

2a

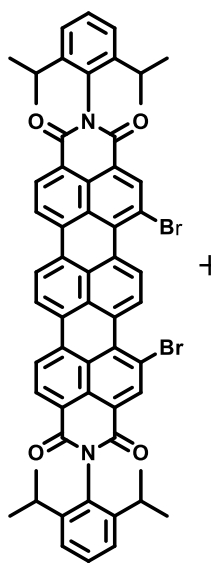

$2 \mathbf{b}$

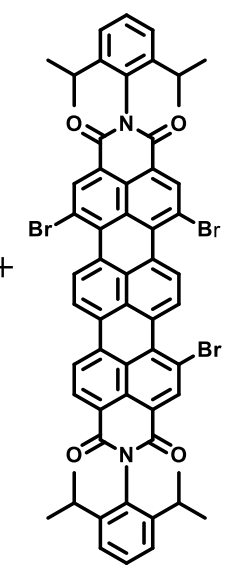

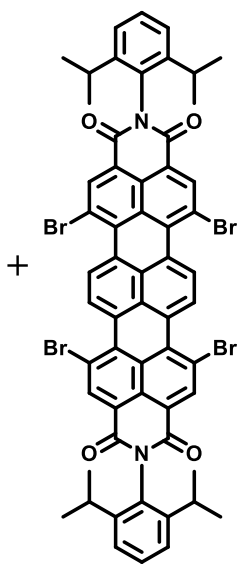

Procedure for Bromination of TDI (1-Br to 4) at different temperature and equivalents: In a dry $250 \mathrm{~mL}$ sealed round bottom flask the mixture of $1(0.12 \mathrm{mmol}, 100 \mathrm{mg})$ and elemental bromine in $100 \mathrm{~mL}$ chloroform was stirred for 2 hours. The remaining bromine was quenched with saturated $\mathrm{Na}_{2} \mathrm{SO}_{3}$ solution. The organic phase was extracted with chloroform and dried over anhydrous $\mathrm{Na}_{2} \mathrm{SO}_{4}$, then concentrated via rotary evaporation. The residue was purified by repetitive column chromatography using basic alumina and neutral alumina as stationary phases and dichloromethane/hexane as eluent (60:40 v/v) to give desired blue solid products.

With 2 Equivalents of Bromine: $(0.24 \mathrm{mmol}, 13.50 \mu \mathrm{L})$

At $0{ }^{\circ} \mathrm{C}$ : 1 - Unreacted (55\%), 1-Br- $49 \mathrm{mg}(45 \%)$

At $27{ }^{\circ} \mathrm{C}$ : 1- Unreacted (48\%), 1-Br- $35 \mathrm{mg}(32 \%)$, 2a- $23 \mathrm{mg}(20 \%)$

At $60{ }^{\circ} \mathrm{C}$ : 1- Unreacted (25\%), 1-Br- $27 \mathrm{mg}(25 \%), 2 \mathbf{a}-33 \mathrm{mg}(28 \%), 2 \mathbf{b}$ - Trace, 3- Trace

With 4 Equivalents of Bromine: $(0.49 \mathrm{mmol}, 25 \mu \mathrm{L})$

At $0{ }^{\circ} \mathrm{C}$ : 1- Unreacted (10\%), 1-Br- $42 \mathrm{mg}(38 \%), 2 a-38 \mathrm{mg}(32 \%), \mathbf{2 b}-$ Trace, $\mathbf{3}$ - Trace

At $27^{\circ} \mathrm{C}$ : 1-Br- $11 \mathrm{mg}(10 \%), \mathbf{2 a}-50 \mathrm{mg}(42 \%), 2 \mathbf{b}-33 \mathrm{mg}(28 \%), 3-25 \mathrm{mg}(20 \%)$

At $60{ }^{\circ} \mathrm{C}$ : 2a- $30 \mathrm{mg}(25 \%), 2 \mathbf{b}-16 \mathrm{mg}(14 \%), 3-56 \mathrm{mg}(44 \%), 4-23 \mathrm{mg}(17 \%)$

Optimized procedure for Bromination of TDI (2-3): In a dry $250 \mathrm{~mL}$ sealed round bottom flask, a mixture of $\mathbf{1}$ (0.12 $\mathrm{mmol}, 100 \mathrm{mg}$ ) and elemental bromine $(0.48 \mathrm{mmol}, 25 \mu \mathrm{L})$ in $100 \mathrm{~mL}$ chloroform was stirred at room temperature for 2 hours. After the completion of the reaction, the remaining bromine was quenched with saturated, aqueous $\mathrm{Na}_{2} \mathrm{SO}_{3}$ solution. The organic phase was extracted with chloroform and dried over anhydrous $\mathrm{Na}_{2} \mathrm{SO}_{4}$, concentrated in a rotary 
evaporator. The residue was purified by repetitive column chromatography using basic alumina and neutral alumina as stationary phase and dichloromethane/hexane as eluent $(60: 40 \mathrm{v} / \mathrm{v})$ to give desired blue solid products. $2 \mathrm{a}: 50 \mathrm{mg}$ (42\%), 2b: $34 \mathrm{mg}(28 \%), 3: 25 \mathrm{mg}(20 \%)$.

2a in $\mathrm{CDCl}_{3}:{ }^{1} \mathrm{H}$ NMR $\left(700 \mathrm{MHz}, \mathrm{CDCl}_{3}, 298 \mathrm{~K}\right) \delta 9.79(\mathrm{~d}, J=8.3 \mathrm{~Hz}, 1 \mathrm{H}), 9.52(\mathrm{~d}, J=8.3 \mathrm{~Hz}, 1 \mathrm{H}), 9.02(\mathrm{~s}, 1 \mathrm{H}), 9.0(\mathrm{~s}, 1 \mathrm{H})$, $8,78(\mathrm{dd}, J=7.8,4.4 \mathrm{~Hz}, 2 \mathrm{H}), 8.76-8.72(\mathrm{~m}, 2 \mathrm{H}), 8.72-8.67(\mathrm{~m}, 2 \mathrm{H}), 7.51$ (ddd, $J=12.7,8.0,4.7 \mathrm{~Hz}, 2 \mathrm{H}), 7.37$ (d, $J=7.9$ $\mathrm{Hz}, 4 \mathrm{H}), 2.76(\mathrm{~m}, 4 \mathrm{H}), 1.20(\mathrm{dd}, J=6.5,5.3 \mathrm{~Hz}, 24 \mathrm{H}) .{ }^{13} \mathrm{C}\left\{{ }^{1} \mathrm{H}\right\} \mathrm{NMR}\left(175 \mathrm{MHz}, \mathrm{CDCl}_{3}, 298 \mathrm{~K}\right) \delta 163.9,163.6,162.8,162,6$, $145.8,145.7,145.7,139.4,138.5,136.0,135.5,135.3,134.7,132.2,131.8,131.4,130.9,130.7,130.7,130.6,130.5,130.4$, $130.0,129.9,129.6,129.4,129.4,129.3,128.9,129.8,127.5,127.0,126.5,124.3,124.3,124.2,123.3,122.9,122.9,122.4$, 122.0, 121.8, 121.3, 120.0, 119.4, 29.8, 29.8, 24.1, 24.1. HRMS (APCI/Q-TOF) $m / z:[M+H]]^{+}$Calculated for $\mathrm{C}_{58} \mathrm{H}_{45} \mathrm{Br}_{2} \mathrm{~N}_{2} \mathrm{O}_{4}$, 993.1728; Found 993.1732.

2a in $\mathbf{C}_{6} \mathbf{D}_{6}$ : ${ }^{1} \mathrm{H}$ NMR $\left(500 \mathrm{MHz}, \mathrm{C}_{6} \mathrm{D}_{6}, 298 \mathrm{~K}\right) \delta 9.43(\mathrm{~d}, J=8.3 \mathrm{~Hz}, 1 \mathrm{H}), 9.15(\mathrm{~d}, J=8.3 \mathrm{~Hz}, 1 \mathrm{H}), 9.11(\mathrm{~s}, 1 \mathrm{H}), 9.03(\mathrm{~s}, 1 \mathrm{H})$, $8.76(\mathrm{~d}, J=7.7 \mathrm{~Hz}, 1 \mathrm{H}), 8.69(\mathrm{~d}, J=7.9 \mathrm{~Hz}, 1 \mathrm{H}), 7.96(\mathrm{~d}, J=8.0 \mathrm{~Hz}, 1 \mathrm{H}), 7.86(\mathrm{~d}, J=8.2 \mathrm{~Hz}, 2 \mathrm{H}), 7.78(\mathrm{~d}, J=8.5 \mathrm{~Hz}, 1 \mathrm{H}), 7.39$ $(\mathrm{t}, J=7.8 \mathrm{~Hz}, 2 \mathrm{H}), 7.30(\mathrm{~d}, J=7.5 \mathrm{~Hz}, 4 \mathrm{H}), 3.10(\mathrm{dd}, J=13.9,6.9 \mathrm{~Hz}, 4 \mathrm{H}), 1.32(\mathrm{~d}, J=6.4 \mathrm{~Hz}, 24 \mathrm{H})$.

2b in CDCl $_{3}{ }^{1} \mathrm{H}$ NMR $\left(700 \mathrm{MHz}, \mathrm{CDCl}_{3}, 298 \mathrm{~K}\right) \delta 9.75(\mathrm{~s}, 2 \mathrm{H}), 9.02(\mathrm{~s}, 2 \mathrm{H}), 8.78(\mathrm{~d}, J=7.9 \mathrm{~Hz}, 2 \mathrm{H}), 8.72(\mathrm{~s}, 2 \mathrm{H}), 8.66(\mathrm{~d}, J$ $=8.0 \mathrm{~Hz}, 2 \mathrm{H}), 7.51(\mathrm{t}, J=7.9 \mathrm{~Hz}, 2 \mathrm{H}), 7.37(\mathrm{~d}, J=7.9 \mathrm{~Hz}, 4 \mathrm{H}), 2.76(\mathrm{dt}, J=13.6,6.8 \mathrm{~Hz}, 4 \mathrm{H}), 1.20(\mathrm{dd}, J=6.6,4.9 \mathrm{~Hz}, 24 \mathrm{H})$. ${ }^{13} \mathrm{C}\left\{{ }^{1} \mathrm{H}\right\}$ NMR $\left(175 \mathrm{MHz}, \mathrm{CDCl}_{3}, 298 \mathrm{~K}\right) \delta 163.6,162.8,145.7,139.4,135.6,135.1,131.8,130.6,130.4,129.9,129.2,128.9$, 128.8, 128.7, 128.3, 124.3, 122.7, 121.9, 121.9, 119.8, 29.9, 29.3, 24.1, 24.1. HRMS (APCI/Q-TOF) $m / z:[M+H]^{+}$ Calculated for $\mathrm{C}_{58} \mathrm{H}_{45} \mathrm{Br}_{2} \mathrm{~N}_{2} \mathrm{O}_{4}$, 993.1728; Found 993.1720.

2b in $\mathbf{C}_{6} \mathbf{D}_{6}$ : ${ }^{1} \mathrm{H}$ NMR $\left(500 \mathrm{MHz}, \mathrm{C}_{6} \mathrm{D}_{6}, 298 \mathrm{~K}\right) \delta 9.41(\mathrm{~s}, 2 \mathrm{H}), 9.07(\mathrm{~s}, 2 \mathrm{H}), 8.69(\mathrm{~d}, J=7.9 \mathrm{~Hz}, 2 \mathrm{H}), 7.90(\mathrm{~d}, J=8.1 \mathrm{~Hz}, 2 \mathrm{H})$, $7.88(\mathrm{~s}, 2 \mathrm{H}), 7.40-7.37(\mathrm{~m}, 2 \mathrm{H}), 7.30(\mathrm{~d}, J=7.8 \mathrm{~Hz}, 4 \mathrm{H}), 3.10(\mathrm{dt}, J=13.6,6.8 \mathrm{~Hz}, 4 \mathrm{H}), 1.33(\mathrm{~d}, J=6.8 \mathrm{~Hz}, 24 \mathrm{H})$.

3 in CDCl $_{3}{ }^{1} \mathrm{H}$ NMR $\left(700 \mathrm{MHz}, \mathrm{CDCl}_{3}, 298 \mathrm{~K}\right) \delta 9.84(\mathrm{~d}, J=8.5 \mathrm{~Hz}, 1 \mathrm{H}), 9.55-9.51(\mathrm{~m}, 2 \mathrm{H}), 9.02(\mathrm{~s}, 1 \mathrm{H}), 9.00(\mathrm{~d}, J=4.5$ $\mathrm{Hz}, 2 \mathrm{H}), 8.79(\mathrm{~d}, J=7.8 \mathrm{~Hz}, 1 \mathrm{H}), 8.69(\mathrm{~d}, J=6.8 \mathrm{~Hz}, 2 \mathrm{H}), 7.52(\mathrm{dd}, J=11.9,7.7 \mathrm{~Hz}, 2 \mathrm{H}), 7.37(\mathrm{~d}, J=7.9 \mathrm{~Hz}, 4 \mathrm{H}), 2.80-2.71$ $(\mathrm{m}, 4 \mathrm{H}), 1.20(\mathrm{~d}, J=6.4 \mathrm{~Hz}, 24 \mathrm{H}) .{ }^{13} \mathrm{C}\left\{{ }^{1} \mathrm{H}\right\}$ NMR $\left(175 \mathrm{MHz}, \mathrm{CDCl}_{3}, 298 \mathrm{~K}\right) \delta 163.6,162.8,162.6,145.8,145.7,139.6,138.5$, $138.5,135.4,135.0,134.8,135.4,131.8,130.5,130.4,130.3,129.3,129.3,128.4,128.1,127.9,127.0,124.3,124.3,123.1$, $122.9,122.1,122.0,121.6,121.4,120.4,120.2,119.9,29.8,29.4,29.3,24.2,24.1$. HRMS (APCI/Q-TOF) $m / z:[M+H]^{+}$ Calculated for $\mathrm{C}_{58} \mathrm{H}_{44} \mathrm{Br}_{3} \mathrm{~N}_{2} \mathrm{O}_{4}, 1071.0831$; Found 1071.0829.

3 in $\mathbf{C}_{6} \mathrm{D}_{6}:{ }^{1} \mathrm{H}$ NMR $\left(500 \mathrm{MHz}, \mathrm{C}_{6} \mathrm{D}_{6}, 298 \mathrm{~K}\right) \delta 9.53(\mathrm{~d}, J=8.6 \mathrm{~Hz}, 1 \mathrm{H}), 9.21(\mathrm{~d}, J=8.6 \mathrm{~Hz}, 1 \mathrm{H}), 9.17(\mathrm{~d}, J=8.4 \mathrm{~Hz}, 1 \mathrm{H}), 9.09$ $(\mathrm{s}, 1 \mathrm{H}), 9.03(\mathrm{~s}, 1 \mathrm{H}), 8.99(\mathrm{~s}, 1 \mathrm{H}), 8.69(\mathrm{~d}, J=7.9 \mathrm{~Hz}, 1 \mathrm{H}), 7.87(\mathrm{~d}, J=8.1 \mathrm{~Hz}, 1 \mathrm{H}), 7.82(\mathrm{~d}, J=8.5 \mathrm{~Hz}, 1 \mathrm{H}), 7.38(\mathrm{dd}, J=$ 11.4, $4.2 \mathrm{~Hz}, 2 \mathrm{H}), 7.29$ (d, $7.9 \mathrm{~Hz}, 4 \mathrm{H}), 3.12-3.03(\mathrm{~m}, 4 \mathrm{H}), 1.31$ (d, $J=6.8,24 \mathrm{H})$.

Procedure for Preparation of 5a: $\mathrm{Pd}\left(\mathrm{PPh}_{3}\right)_{2} \mathrm{Cl}_{2}$ (0.1 equiv.), CuI (catalytic) and $\mathbf{2 a}(0.05 \mathrm{mmol}, 50 \mathrm{mg})$ were added into an oven-dried, argon-flushed Schlenk flask. Dry toluene (5 mL) and distilled triethylamine (5 mL) were injected into the flask under argon atmosphere and the resulting mixture was degassed for 30 minutes. Ethynylferrocene $(0.15$ mmol, $32 \mathrm{mg}$ ) was added and the reaction mixture was stirred at $85^{\circ} \mathrm{C}$ using an oil bath for 16 hours under argon. The completion of reaction was monitored by thin layer chromatography. After cooling to room temperature, it was concentrated in a rotary evaporator. The residue was purified by silica gel (mess 100-200) column chromatography (dichloromethane/hexane) to give the desired product as a green solid (48 mg, $76 \%$ ). ${ }^{1} \mathrm{H} \mathrm{NMR} \mathrm{(500} \mathrm{MHz,} \mathrm{C}_{6} \mathrm{D}_{6}, 298 \mathrm{~K}$ ) $\delta 10.14(\mathrm{~d}, J=8.4 \mathrm{~Hz}, 2 \mathrm{H}), 9.26(\mathrm{~s}, 2 \mathrm{H}), 8.71(\mathrm{~d}, J=8.0 \mathrm{~Hz}, 2 \mathrm{H}), 8.29(\mathrm{~d}, J=8.7 \mathrm{~Hz}, 2 \mathrm{H}), 8.05(\mathrm{~d}, J=8.2 \mathrm{~Hz}, 2 \mathrm{H}), 7.37(\mathrm{~d}, J$ $=7.3 \mathrm{~Hz}, 2 \mathrm{H}), 7.31-7.28(\mathrm{~m}, 4 \mathrm{H}), 4.63(\mathrm{~s}, 4 \mathrm{H}), 4.13(\mathrm{~s}, 10 \mathrm{H}), 4.11(\mathrm{~s}, 4 \mathrm{H}), 3.15-3.08(\mathrm{~m}, 4 \mathrm{H}), 1.31(\mathrm{~d}, J=6.8 \mathrm{~Hz}, 24 \mathrm{H})$. ${ }^{13} \mathrm{C}\left\{{ }^{1} \mathrm{H}\right\}$ NMR $\left(175 \mathrm{MHz}, \mathrm{C}_{6} \mathrm{D}_{6}, 298 \mathrm{~K}\right) \delta 163.6,163.0,146.0,146.0,137.5,135.7,135.0,131.6,130.8,130.7,130.4,128.3$, 128.0, 127.9, 127.3, 126.2, 124.0, 123.9, 123.0, 122.1, 121.5, 121.3, 119.9, 98.1, 88.4, 71.7, 70.3, 70.3, 70.2, 70.2, 69.9, 64.4, 29.8, 29.5, 29.5, 24.0, 23.9. HRMS (APCI/Q-TOF) $m / z$ : $[\mathrm{M}+\mathrm{H}]+$ Calculated for $\mathrm{C}_{82} \mathrm{H}_{63} \mathrm{Fe}_{2} \mathrm{~N}_{2} \mathrm{O}_{4}, 1251.3487$; Found 1251.3476.

Procedure for Preparation of $\mathbf{5 b}$ : $\mathbf{5 b}$ was synthesized using the above procedure with $\mathbf{2 b}(0.05 \mathrm{mmol}, 50 \mathrm{mg})$, ethynylferrocene ( $0.15 \mathrm{mmol}, 32 \mathrm{mg})$, and obtained as a green solid (45 mg, $\left.71 \%) .{ }^{1} \mathrm{H} \mathrm{NMR} \mathrm{(500} \mathrm{MHz,} \mathrm{CDCl} 3,298 \mathrm{~K}\right) \delta$ $10.34(\mathrm{~s}, 2 \mathrm{H}), 8.86(\mathrm{~s}, 2 \mathrm{H}), 8.74(\mathrm{~d}, J=8.5 \mathrm{~Hz}, 2 \mathrm{H}), 8.73(\mathrm{~s}, 2 \mathrm{H}), 8.66(\mathrm{~d}, J=8.2 \mathrm{~Hz}, 2 \mathrm{H}), 7.53(\mathrm{t}, J=7.8 \mathrm{~Hz}, 2 \mathrm{H}), 7.39(\mathrm{~d}, J$ $=7.9 \mathrm{~Hz}, 4 \mathrm{H}), 4.64(\mathrm{~s}, 4 \mathrm{H}), 4.34(\mathrm{~s}, 4 \mathrm{H}), 4.28(\mathrm{~s}, 10 \mathrm{H}), 2.82(\mathrm{~m}, 4 \mathrm{H}), 1.23(\mathrm{~d}, J=6.9 \mathrm{~Hz}, 24 \mathrm{H}) .{ }^{13} \mathrm{C}\left\{{ }^{1} \mathrm{H}\right\} \mathrm{NMR}(175 \mathrm{MHz}$, $\left.\mathrm{CDCl}_{3}, 298 \mathrm{~K}\right) \delta 163.9,163.5,145.8,139.3,131.3,130.8,130.8,129.8,129.0,127.3,124.3,124.2,122.3,121.5,120.9$, 119.5, 100.8, 88.6, 71.9, 70.2, 70.1, 64.1, 29.8, 29.4, 24.2, 24.2 HRMS (APCI/Q-TOF) $\mathrm{m} / \mathrm{z}:[\mathrm{M}+\mathrm{H}]^{+}$Calculated for $\mathrm{C}_{82} \mathrm{H}_{63} \mathrm{Fe}_{2} \mathrm{~N}_{2} \mathrm{O}_{4}, 1251.3487$; Found 1251.3466.

Procedure for Preparation of 6: 6 was synthesized using the above procedure from $3(0.05 \mathrm{mmol}, 50 \mathrm{mg})$, ethynylferrocene $(0.20 \mathrm{mmol}, 42 \mathrm{mg})$, and obtained as a green solid (55 mg, $74 \%) .{ }^{1} \mathrm{H}$ NMR $\left(500 \mathrm{MHz}, \mathrm{CDCl}_{3}, 298 \mathrm{~K}\right) \delta$ $10.48(\mathrm{~d}, J=8.8 \mathrm{~Hz}, 1 \mathrm{H}), 10.24(\mathrm{~d}, J=8.8 \mathrm{~Hz}, 1 \mathrm{H}), 10.18(\mathrm{~d}, J=8.5 \mathrm{~Hz}, 1 \mathrm{H}), 8.94(\mathrm{~d}, J=2.3 \mathrm{~Hz}, 2 \mathrm{H}), 8.92(\mathrm{~s}, 1 \mathrm{H}), 8.83(\mathrm{~d}$, $J=8.6 \mathrm{~Hz}, 1 \mathrm{H}), 8.79(\mathrm{~s}, 2 \mathrm{H}), 7.53(\mathrm{dd}, J=14.9,7.3,2 \mathrm{H}), 7.33(\mathrm{dd}, J=7.8,5.0 \mathrm{~Hz}, 4 \mathrm{H}), 4.7(\mathrm{t}, J=1.7 \mathrm{~Hz}, 2 \mathrm{H}), 4.68-4.67(\mathrm{~m}$, $2 \mathrm{H}), 4.66(\mathrm{~d}, J=1.7 \mathrm{~Hz}, 2 \mathrm{H}), 4.44(\mathrm{~m}, 2 \mathrm{H}), 4.37(\mathrm{~d}, J=1.7 \mathrm{~Hz}, 2 \mathrm{H}), 4.34(\mathrm{~d}, J=4.3 \mathrm{~Hz}, 7 \mathrm{H}), 4.29(\mathrm{~s}, 5 \mathrm{H}), 4.26(\mathrm{~s}, 5 \mathrm{H}), 2.81$ $(\mathrm{m}, 4 \mathrm{H}), 1.23(\mathrm{~d}, J=6.8 \mathrm{~Hz}, 24 \mathrm{H}) .{ }^{13} \mathrm{C}\left\{{ }^{1} \mathrm{H}\right\}$ NMR $\left(175 \mathrm{MHz}, \mathrm{CDCl}_{3}, 298 \mathrm{~K}\right) \delta 163.9,163.5,163.5,145.8,142.9,139.4,139.3$, $138.1,137.8,136.0,135.1,135.0,131.5,131.4,131.2,130.9,130.8,130.7,129.7,127.9,127.6,127.4,126.8,125.7,124.2$, $124.2,121.6,120.9,120.7,120.7,120.2,119.5,118.1,114.2,104.0,100.8,99.0,98.3,88.7,88.3,88.2,81.8,81.4,80.5$, 71.9, 71.9, 71.8, 70.4, 70.4, 70.3, 70.1, 70.0, 32.0, 32.0, 29.8, 29.8, 24.2, 24.2. HRMS (APCI/Q-TOF) $m / z$ : Calculated for $\mathrm{C}_{94} \mathrm{H}_{70} \mathrm{Fe}_{3} \mathrm{~N}_{2} \mathrm{O}_{4}, 1459.3460$; Found 1459.3495.

\section{3: NBO calculations}

Having confirmed the chemical integrity and unambiguous structural elucidation, it became necessary to understand the preference of orientation for the bromine at the bay positions. A thorough investigation on this had been reported by Zhan and Yao on PDI.[1,2] Along the same line, natural bond orbital (NBO) analysis for TDI was carried out (see SI). Even in TDIs, the four bay positions (1-,6-,9- \& 14-) are equally predisposed (-0.212|e|) for ready bromination. 
However, after the monobromination, the NBO analysis suggests that the 6- position $(-0.213|\mathrm{e}|)$ gets activated and the 9- and 14- positions - $0.210 \mid$ e $|\&-0.208|$ e $\mid$ get deactivated. Thus, it can be deduced that the formation of the 1,6dibromo TDI is most favoured and 1,14-dibromo TDI is the least favoured, in contrast with the experimental observation.

Table S1- The charge density at the bay- carbons of 1 and 1-Br.

\begin{tabular}{|l|l|l|l|l|l|}
\hline Comp & $1-$ director & $1-\mathrm{a}$ & $6-$ & $9-$ & 14 \\
\hline 1 & - & $-0.2115^{\mathrm{b}}$ & -0.2115 & -0.2115 & -0.2115 \\
\hline $1-\mathrm{Br}$ & $1-\mathrm{Br}$ & -0.0907 & -0.2131 & -0.2101 & -0.20878 \\
\hline
\end{tabular}

a The 1-position; b NBO (Natural Bond Orbital) charge density, all in $|\mathrm{e}|$. Blue/yellow represents activation/deactivation.[1,2]

1: Zhang, X.; Zhan, C.; Zhang, X.; Yao, J. Orientation of bromination in bay-region of perylene diimides Tetrahedron 2013, 69, $38,8155-8160$.

2: M. J. Frisch, G. W. Trucks, H. B. Schlegel, G. E. Scuseria, M. A. Robb, J. R. Cheeseman, G. Scalmani, V. Barone, B. Mennucci, G. A. Petersson, H. Nakatsuji, M. Caricato, X. Li, H. P. Hratchian, A. F. Izmaylov, J. Bloino, G. Zheng, J. L. Sonnenberg, M. Hada, M. Ehara, K. Toyota, R. Fukuda, J. Hasegawa, M. Ishida, T. Nakajima, Y. Honda, O. Kitao, H. Nakai, T. Vreven, J. A. Montgomery Jr., J. E. Peralta, F. Ogliaro, M. Bearpark, J. J. Heyd, E. Brothers, K. N. Kudin, V. N. Staroverov, R. Kobayashi, J. Normand, K. Raghavachari, A. Rendell, J. C. Burant, S. S. Iyengar, J. Tomasi, M. Cossi, N. Rega, J. M. Millam, M. Klene, J. E. Knox, J. B. Cross, V. Bakken, C. Adamo, J. Jaramillo, R. Gomperts, R. E. Stratmann, O. Yazyev, A. J. Austin, R. Cammi, C. Pomelli, J. W. Ochterski, R. L. Martin, K. Morokuma, V. G. Zakrzewski, G. A. Voth, P. Salvador, J. J. Dannenberg, S. Dapprich, A. D. Daniels, Ö. Farkas, J. B. Foresman, J. V. Ortiz, J. Cioslowski, D. J. Fox, Gaussian 09, Revision D.01, Gaussian, Inc, Wallingford CT, 2013. 
4: NMR spectra

a)

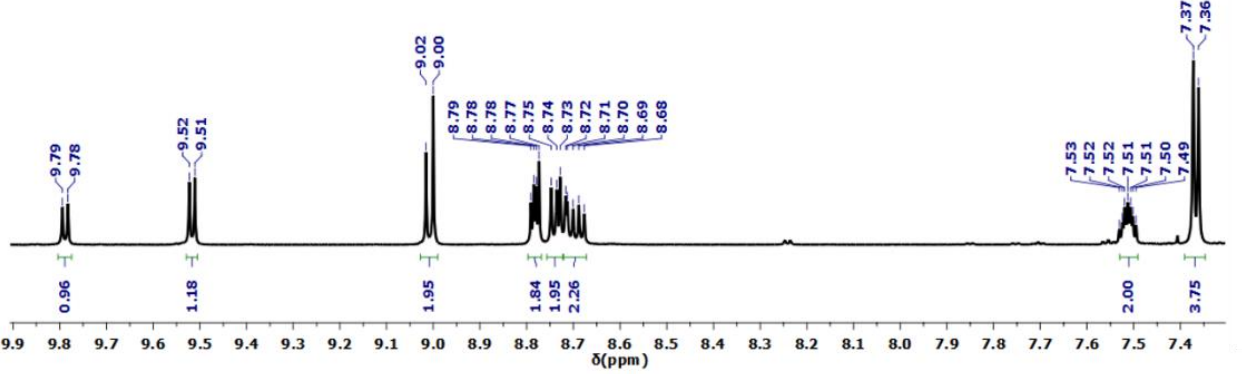

b)

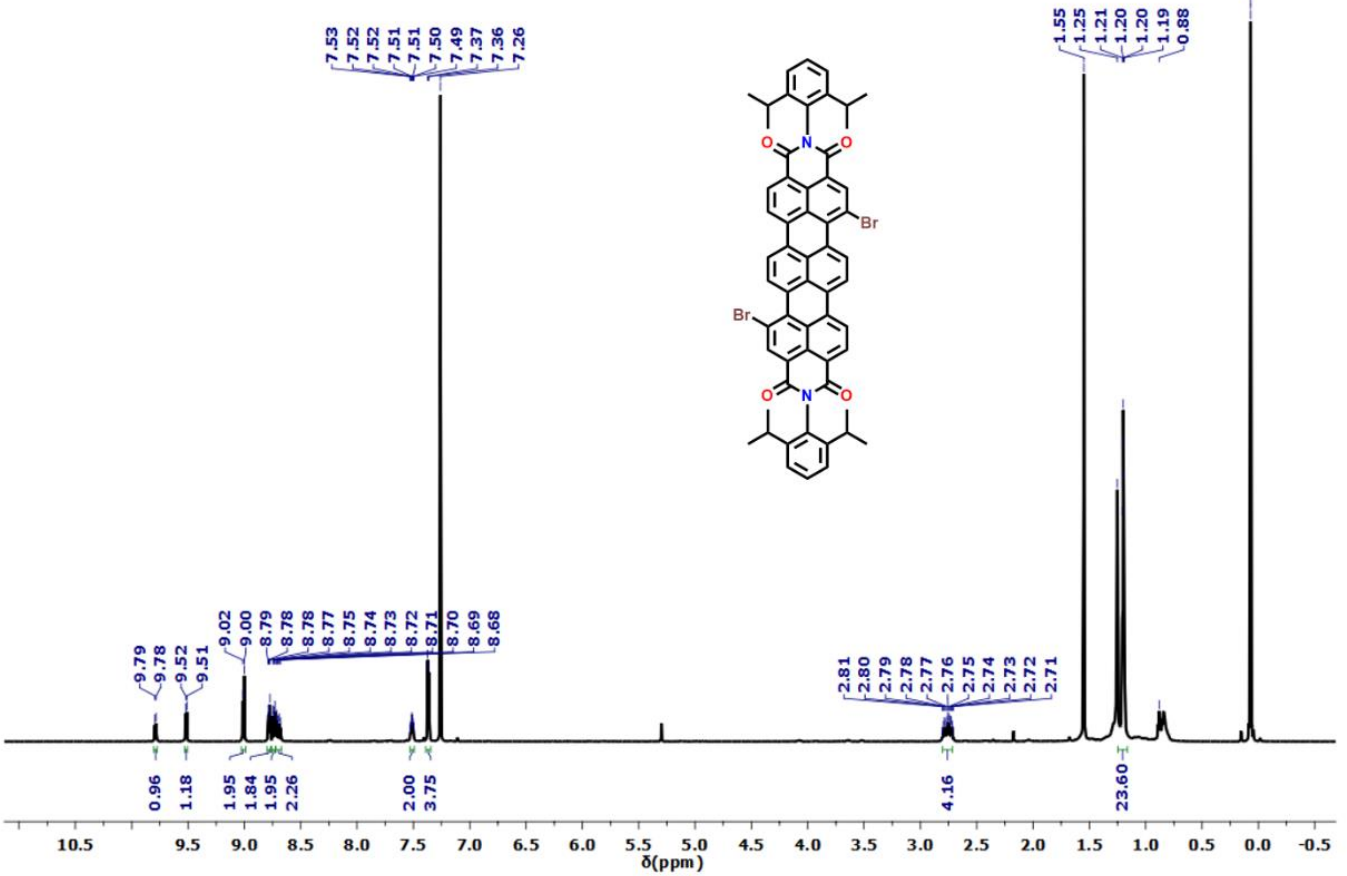

Figure S1: a) zoomed-in and b) full ${ }^{1} \mathrm{H}$ NMR spectra of $\mathbf{2 a}\left(700 \mathrm{MHz}, \mathrm{CDCl}_{3}, 298 \mathrm{~K}\right)$

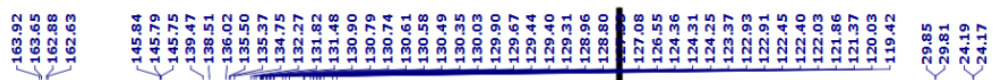
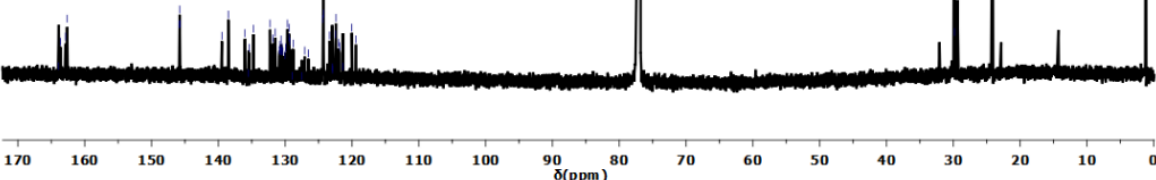

Figure S2: ${ }^{13} \mathrm{C}\left\{{ }^{1} \mathrm{H}\right\}$ NMR spectra of $\mathbf{2 a}\left(175 \mathrm{MHz}, \mathrm{CDCl}_{3}, 298 \mathrm{~K}\right)$ 
a)

c d

h,i
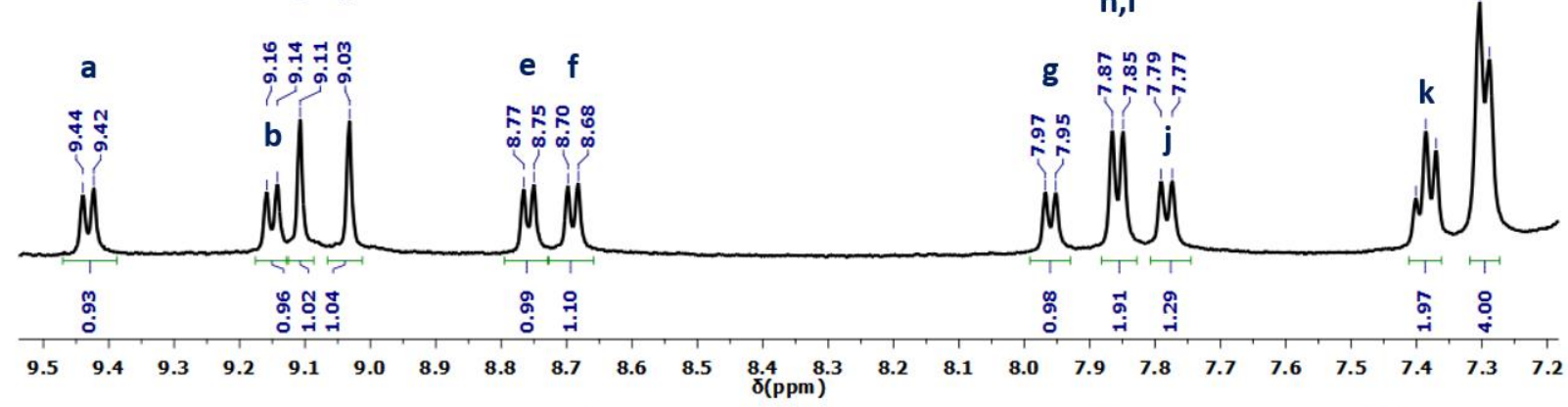

b)

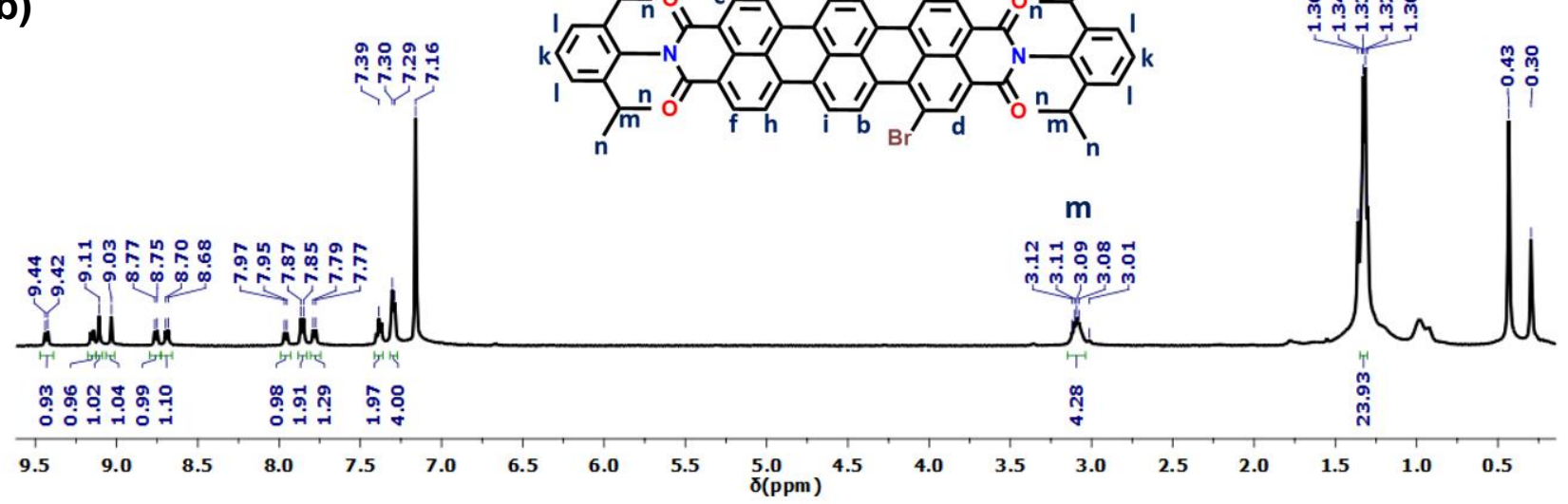

Figure S3: a) zoomed-in and b) full ${ }^{1} \mathrm{H}$ NMR spectra of $\mathbf{2 a}\left(500 \mathrm{MHz}, \mathrm{C}_{6} \mathrm{D}_{6}, 298 \mathrm{~K}\right)$

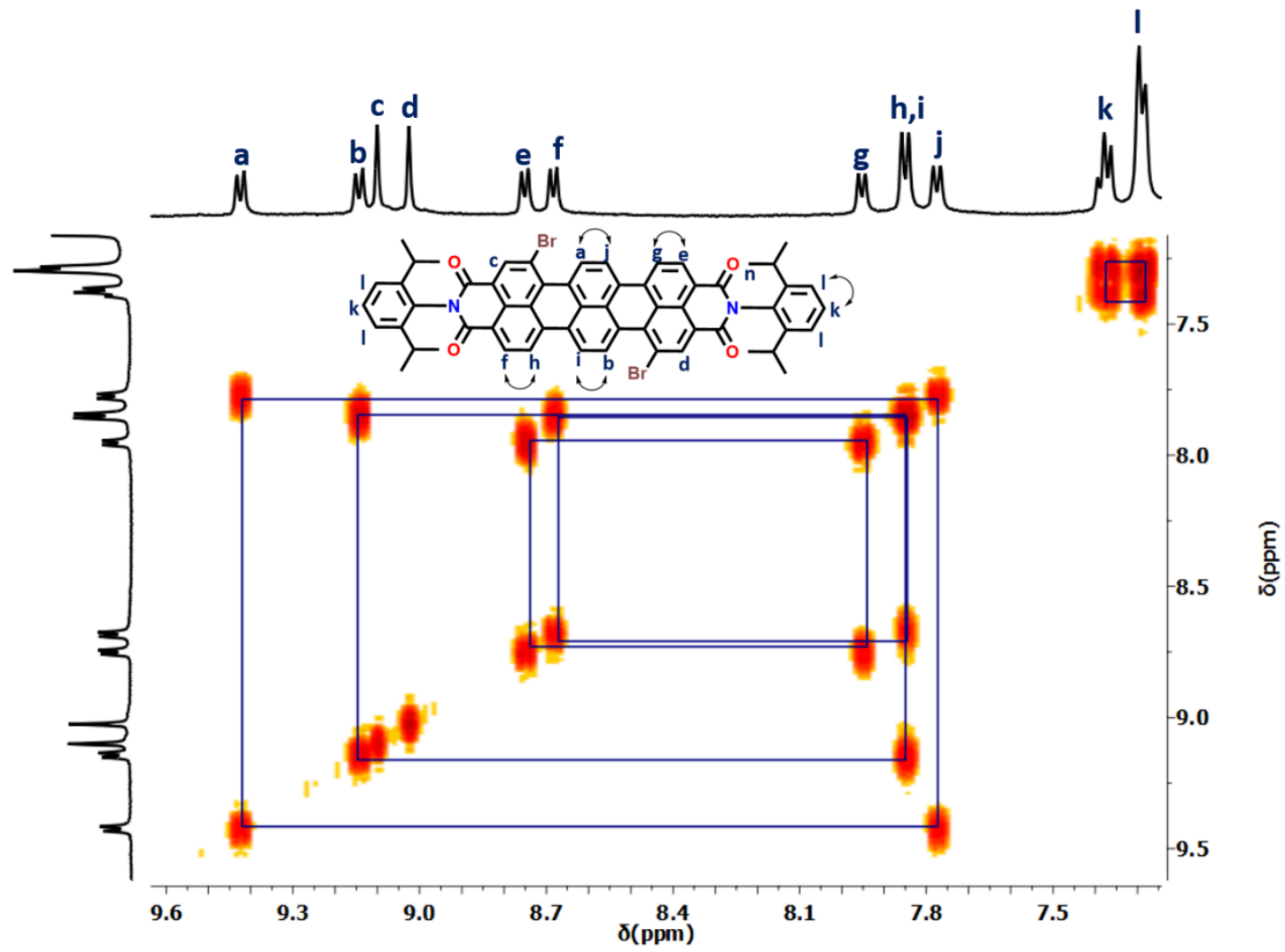

Figure S4: ${ }^{1} \mathrm{H}^{-1} \mathrm{H}$ Correlation (COSY) NMR of $\mathbf{2 a}\left(500 \mathrm{MHz}, \mathrm{C}_{6} \mathrm{D}_{6}, 298 \mathrm{~K}\right)$ 


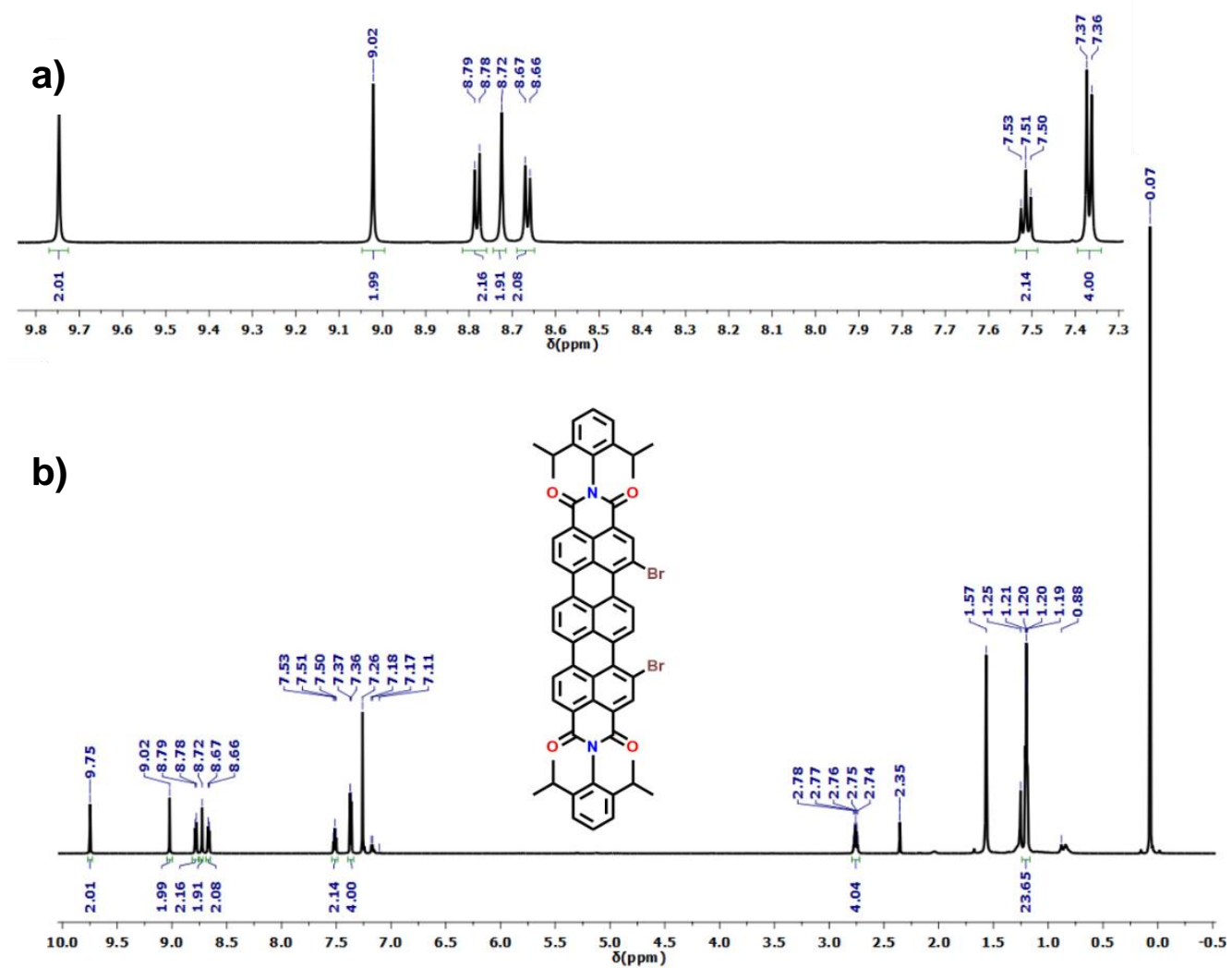

Figure S5: a) zoomed-in and b) full ${ }^{1} \mathrm{H}$ NMR spectra of $\mathbf{2 b}\left(700 \mathrm{MHz}, \mathrm{CDCl}_{3}, 298 \mathrm{~K}\right)$

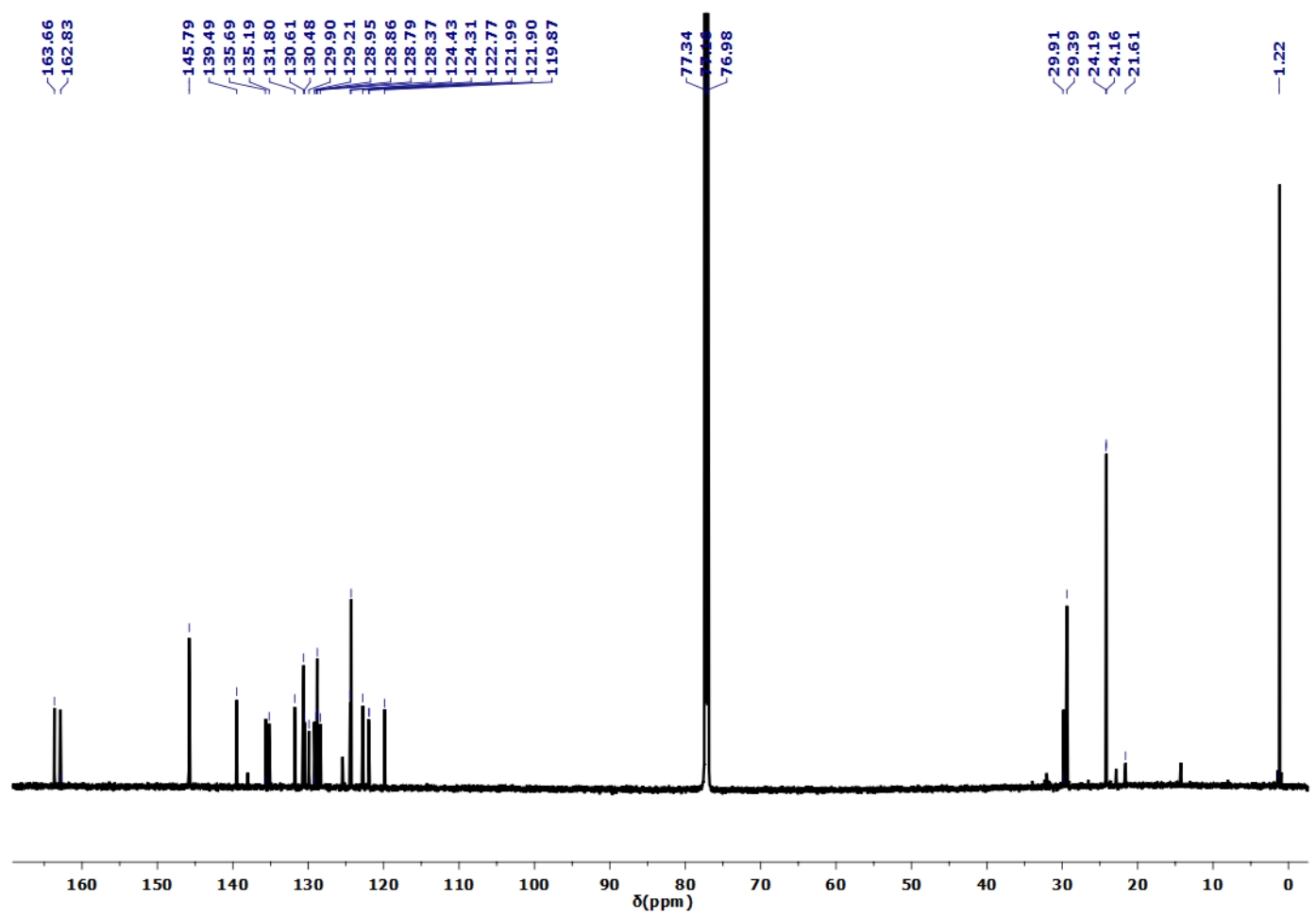

Figure S6: ${ }^{13} \mathrm{C}\left\{{ }^{1} \mathrm{H}\right\}$ NMR spectra of $\mathbf{2 b}\left(175 \mathrm{MHz}, \mathrm{CDCl}_{3}, 298 \mathrm{~K}\right)$ 


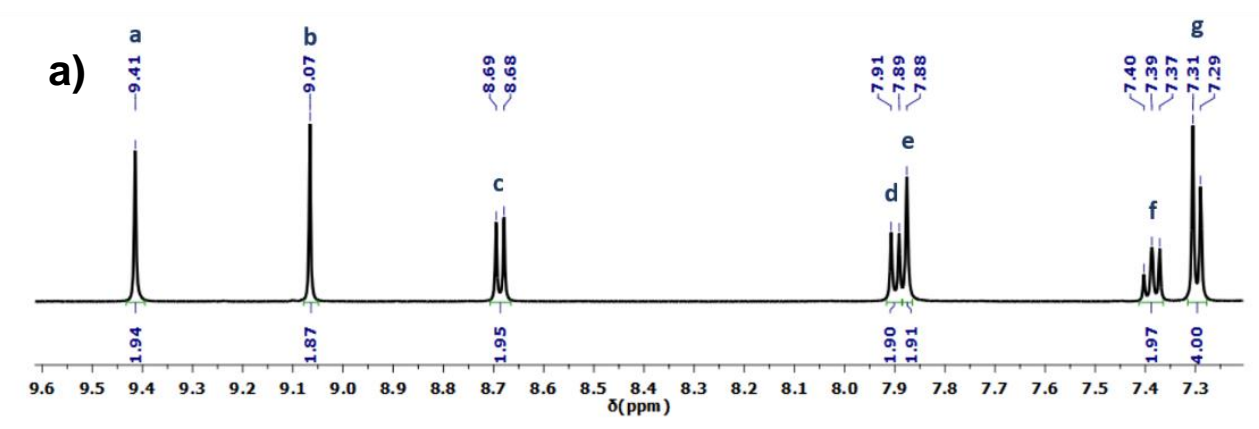

b)
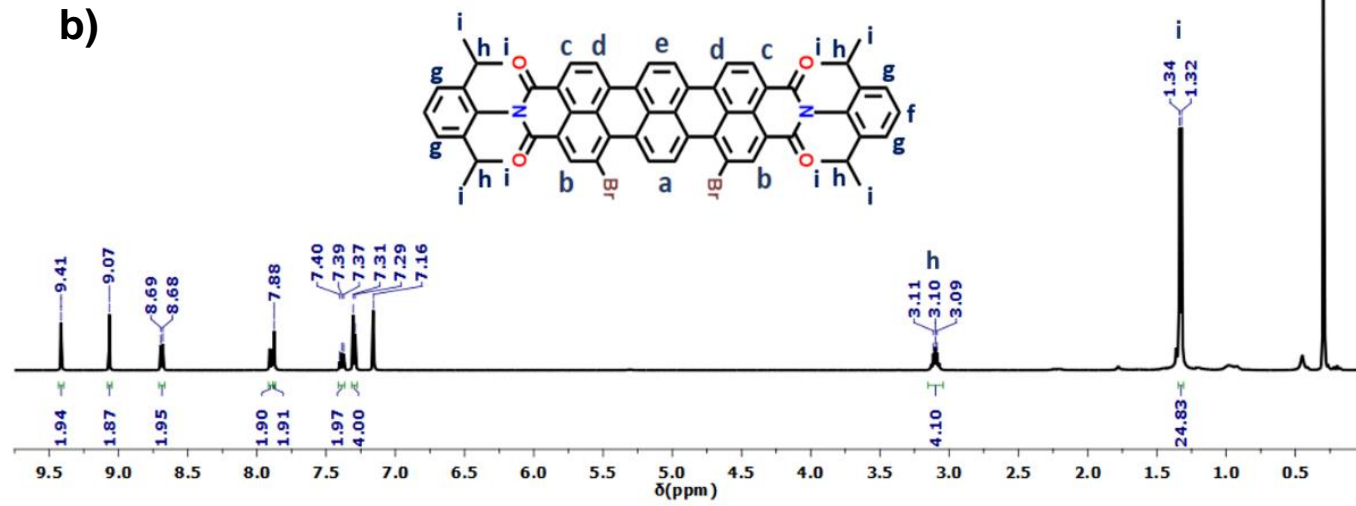

Figure S7: a) zoomed-in and b) full ${ }^{1} \mathrm{H}$ NMR spectra of $\mathbf{2 b}\left(500 \mathrm{MHz}, \mathrm{C}_{6} \mathrm{D}_{6}, 298 \mathrm{~K}\right.$ )

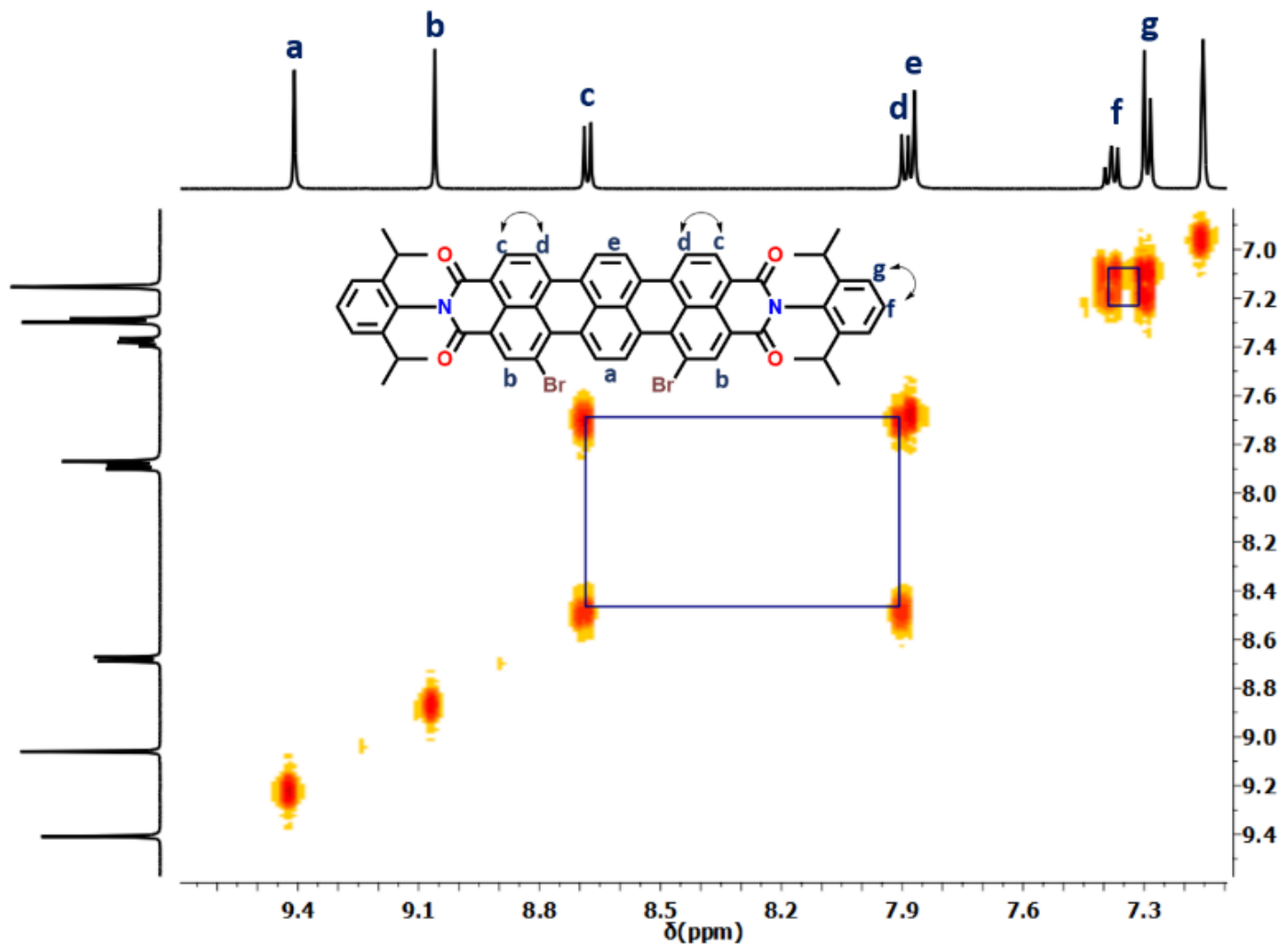

Figure S8: ${ }^{1} \mathrm{H}-1{ }^{-1}$ Correlation (COSY) NMR of $\mathbf{2 b}\left(500 \mathrm{MHz}, \mathrm{C}_{6} \mathrm{D}_{6}, 298 \mathrm{~K}\right)$ 
a)

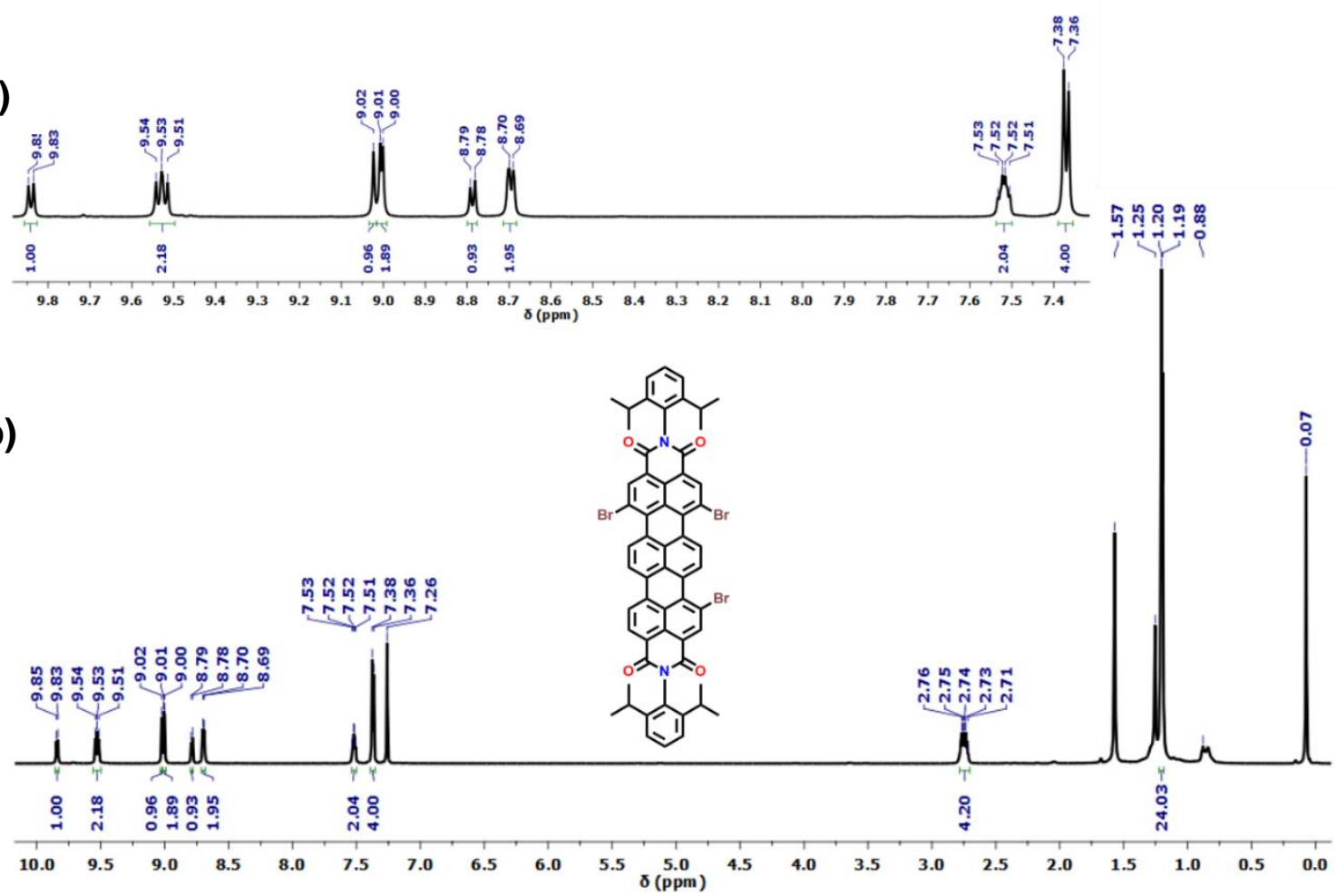

Figure S9: a) zoomed-in and b) full ${ }^{1} \mathrm{H}$ NMR spectra of 3 (700 MHz, $\mathrm{CDCl}_{3}, 298 \mathrm{~K}$ )

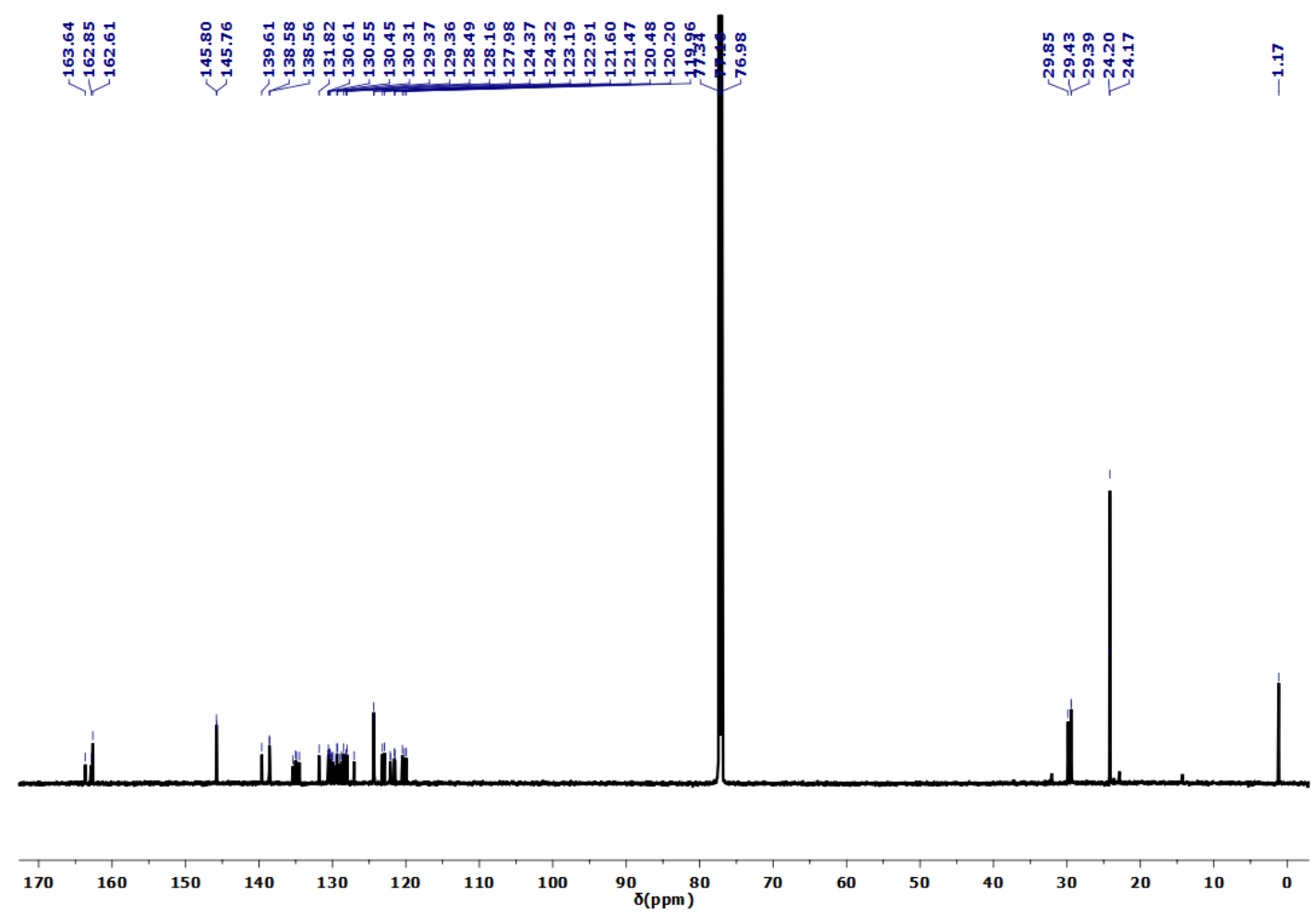

Figure S10: ${ }^{13} \mathrm{C}\left\{{ }^{1} \mathrm{H}\right\}$ NMR spectra of $3\left(175 \mathrm{MHz}, \mathrm{CDCl}_{3}, 298 \mathrm{~K}\right)$ 
a)

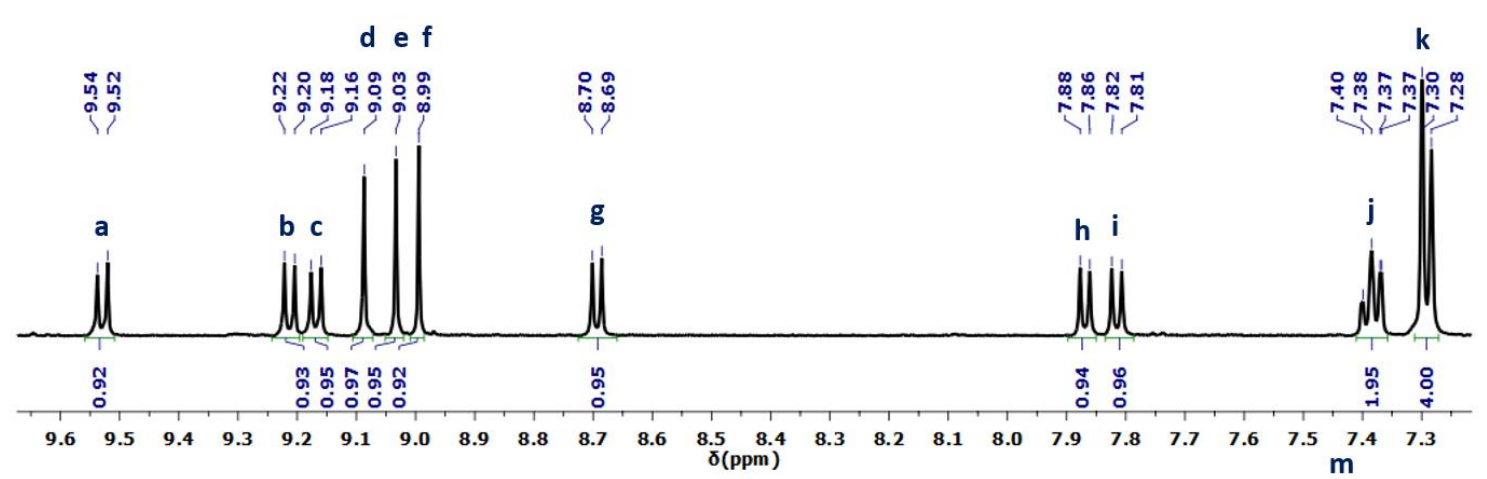

b)

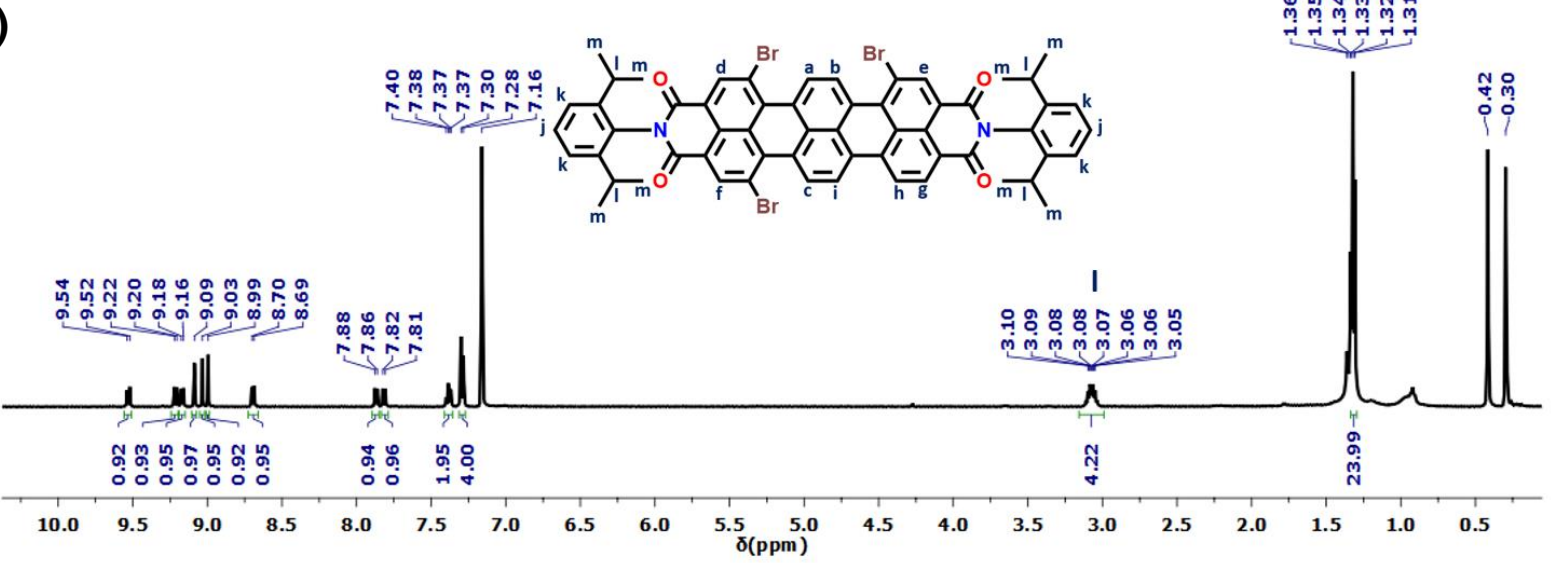

Figure S11: a) zoomed-in and b) full ${ }^{1} \mathrm{H}$ NMR spectra of $3\left(500 \mathrm{MHz}, \mathrm{C}_{6} \mathrm{D}_{6}, 298 \mathrm{~K}\right.$ )

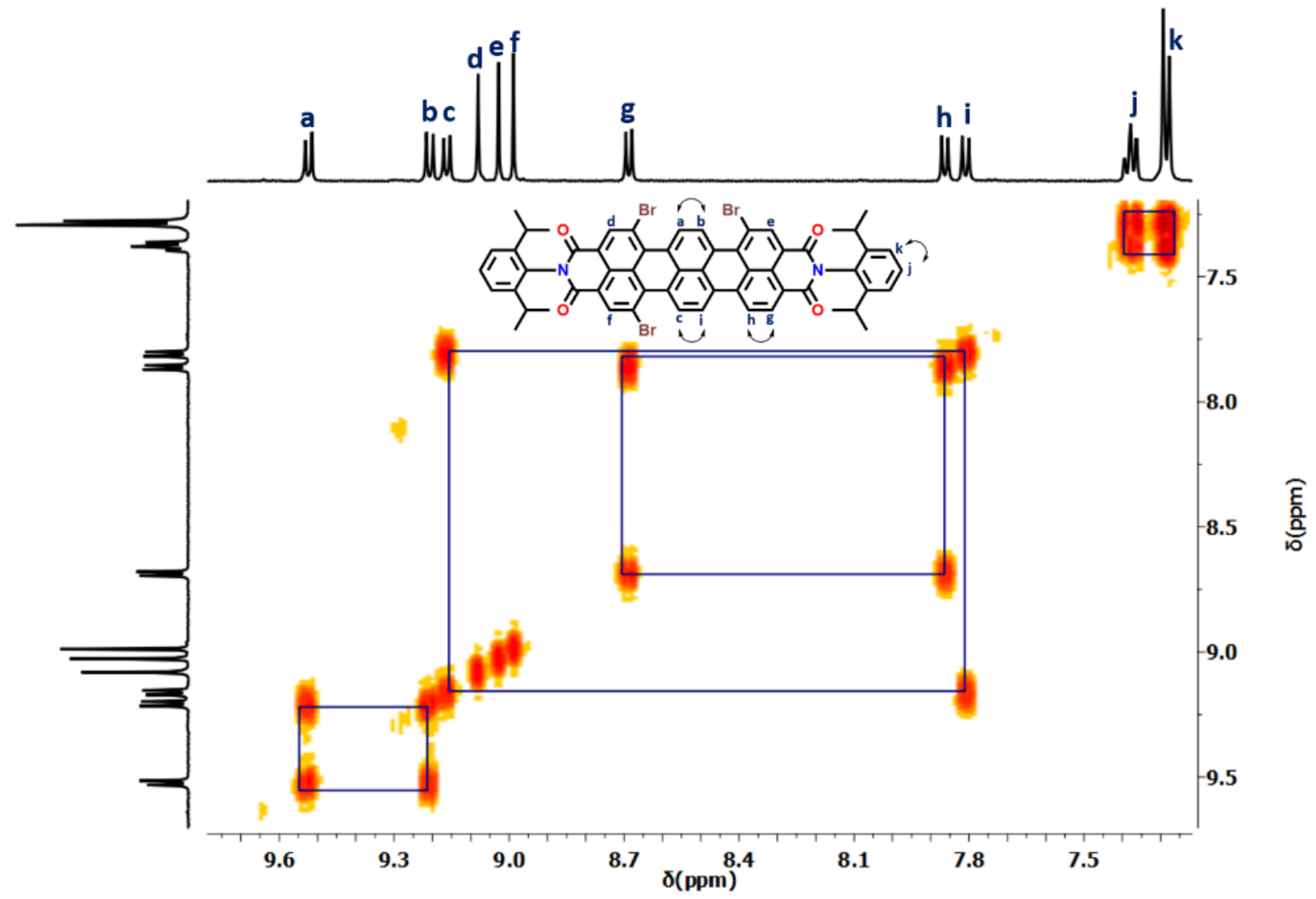

Figure S12: ${ }^{1} \mathrm{H}^{-1} \mathrm{H}$ Correlation (COSY) NMR of 3 (500 MHz, $\left.\mathrm{C}_{6} \mathrm{D}_{6}, 298 \mathrm{~K}\right)$ 
a)

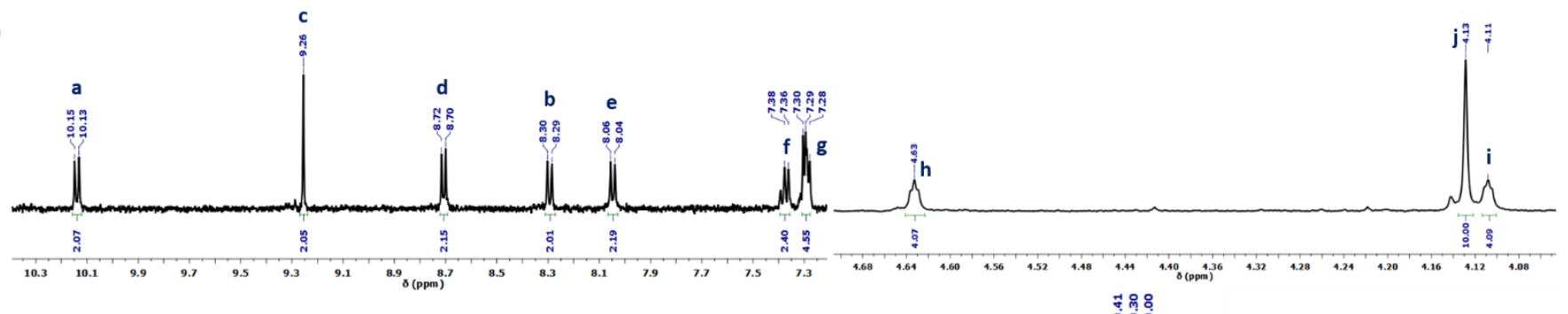

b)

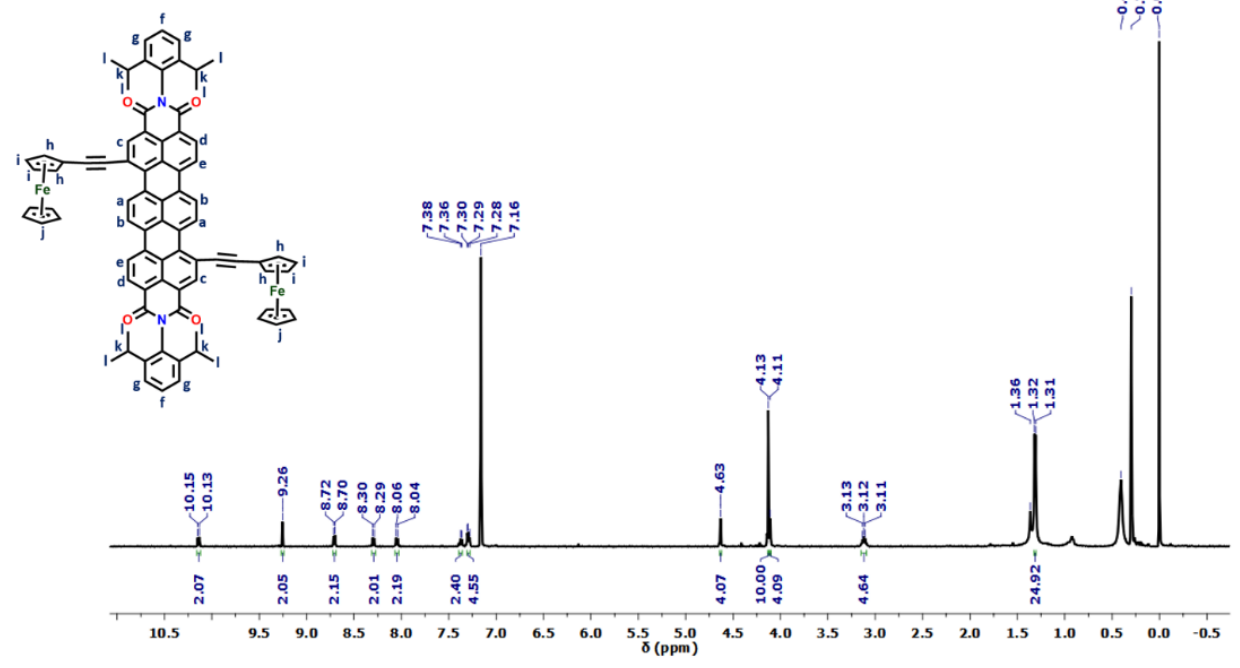

Figure S13: a) zoomed-in and b) full ${ }^{1} \mathrm{H}$ NMR spectra of $\mathbf{5 a}\left(500 \mathrm{MHz}, \mathrm{C}_{6} \mathrm{D}_{6}, 298 \mathrm{~K}\right.$ )

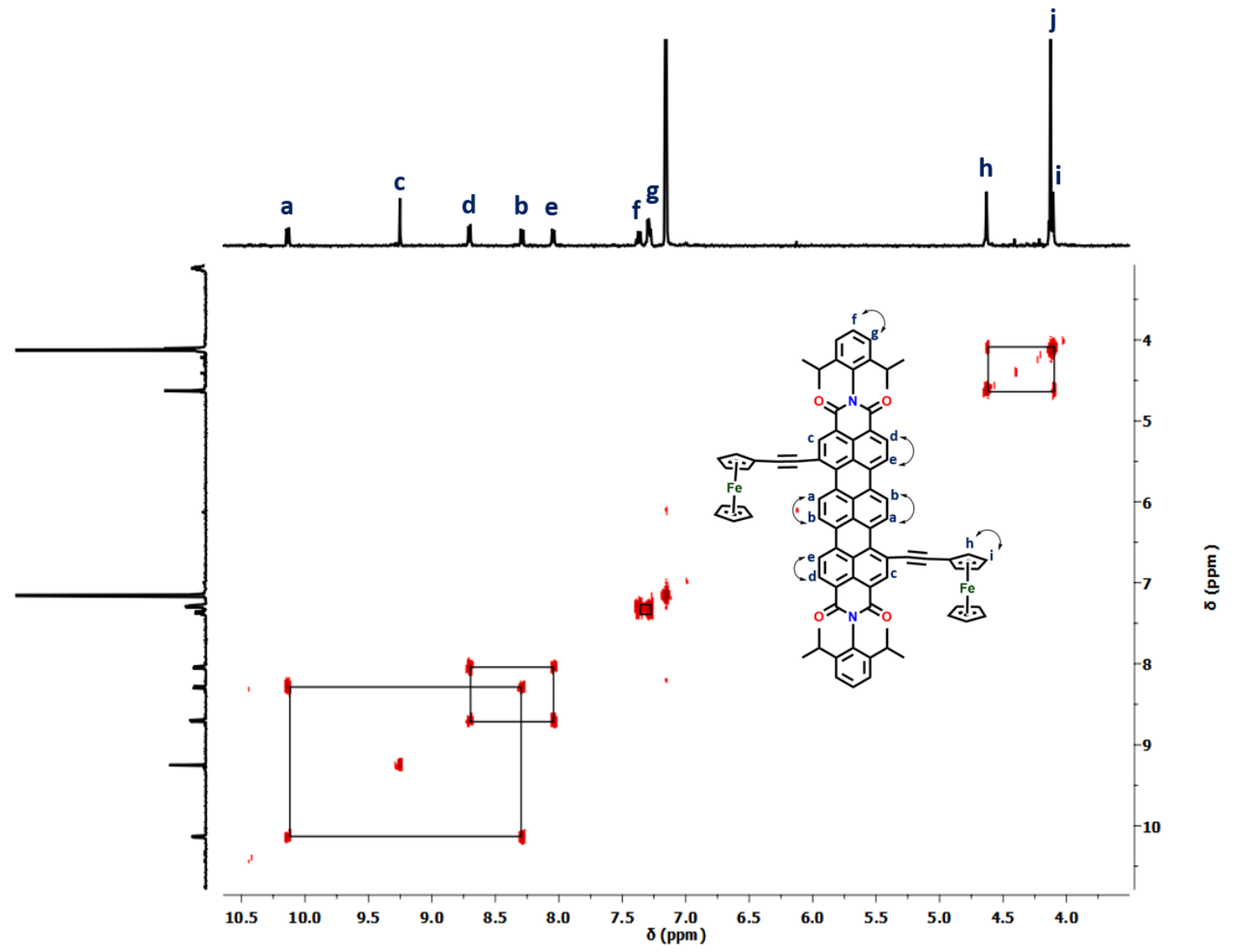

Figure S14: ${ }^{1} \mathrm{H}-1{ }^{1} \mathrm{H}$ Correlation (COSY) NMR of $\mathbf{5 a}\left(500 \mathrm{MHz}, \mathrm{C}_{6} \mathrm{D}_{6}, 298 \mathrm{~K}\right)$ 


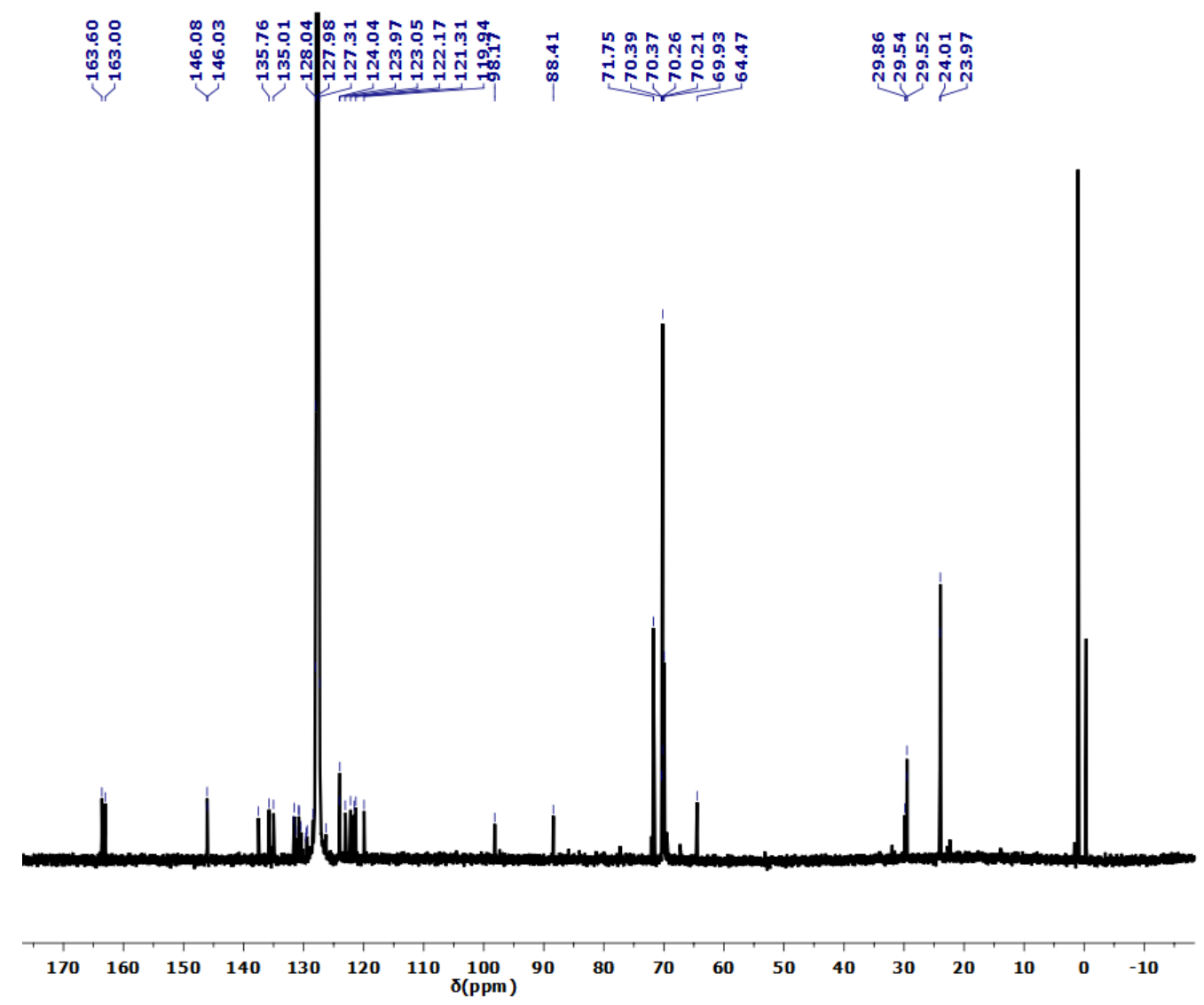

Figure S15: ${ }^{13} \mathrm{C}\left\{{ }^{1} \mathrm{H}\right\} \mathrm{NMR}$ spectra of $\mathbf{5 a}\left(175 \mathrm{MHz}, \mathrm{C}_{6} \mathrm{D}_{6}, 298 \mathrm{~K}\right)$

a)

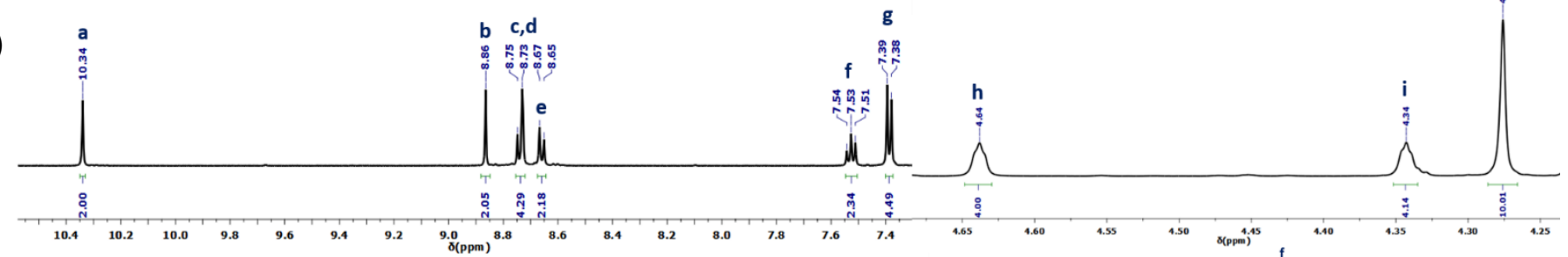

b)

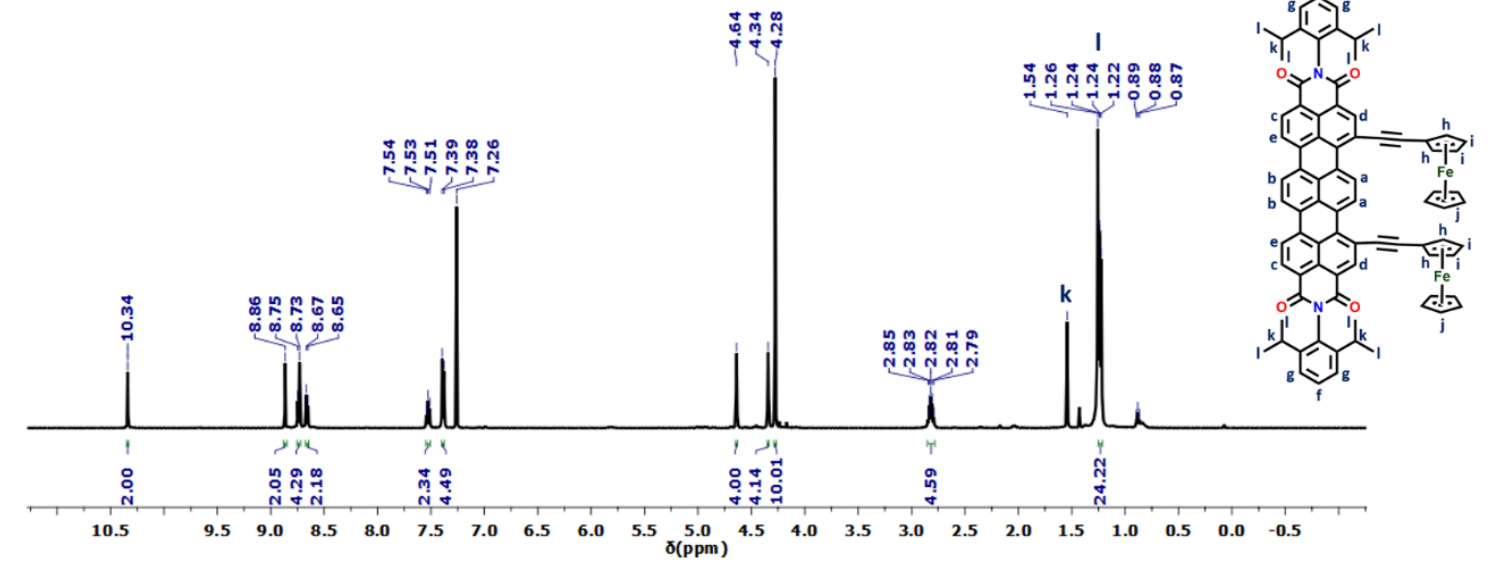

Figure S16: a) zoomed-in and b) full ${ }^{1} \mathrm{H}$ NMR spectra of $\mathbf{5 b}\left(500 \mathrm{MHz}, \mathrm{CDCl}_{3}, 298 \mathrm{~K}\right)$ 


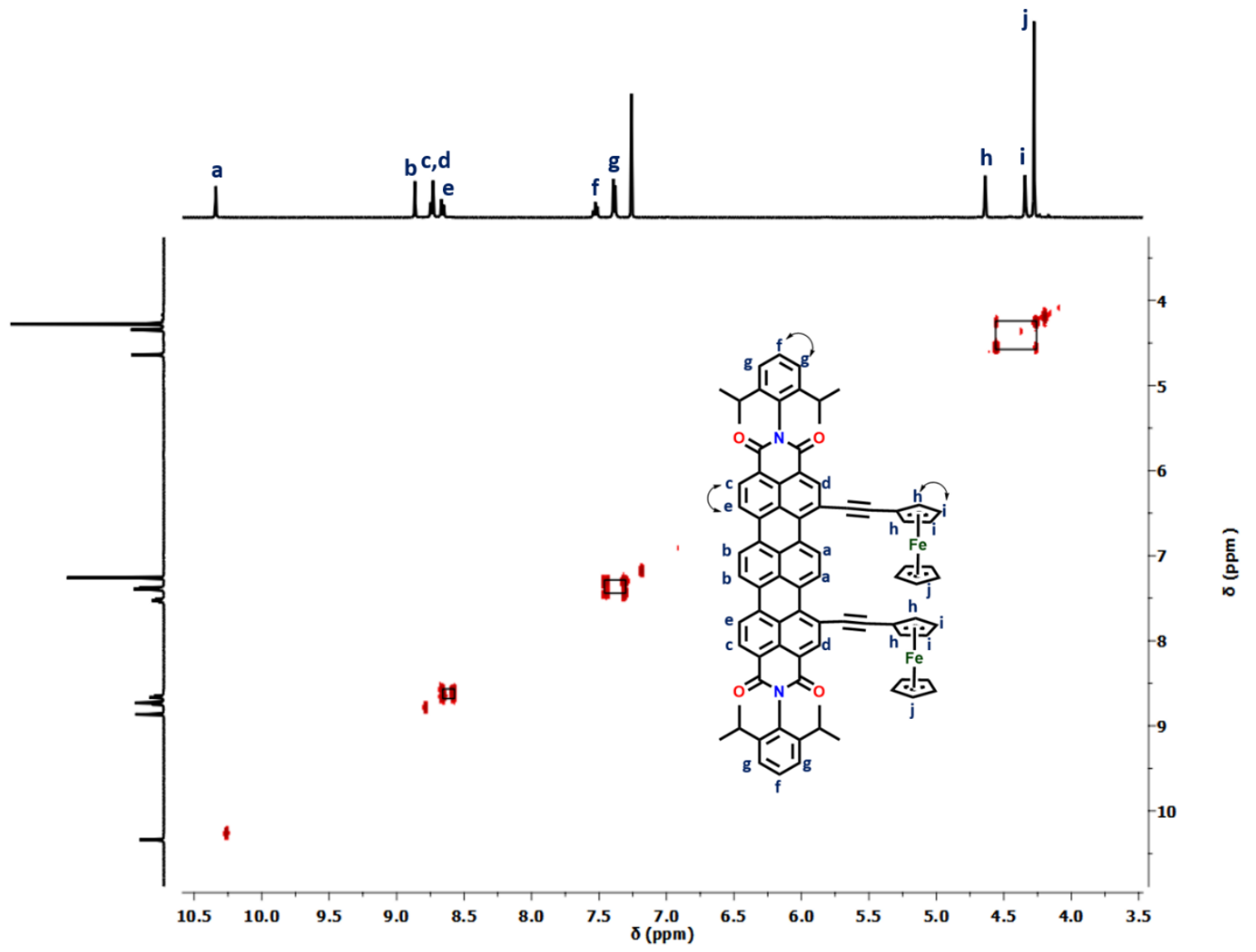

Figure S17: ${ }^{1} \mathrm{H}-1{ }^{-1}$ Correlation (COSY) NMR of $\mathbf{5 b}\left(500 \mathrm{MHz}, \mathrm{CDCl}_{3}, 298 \mathrm{~K}\right)$

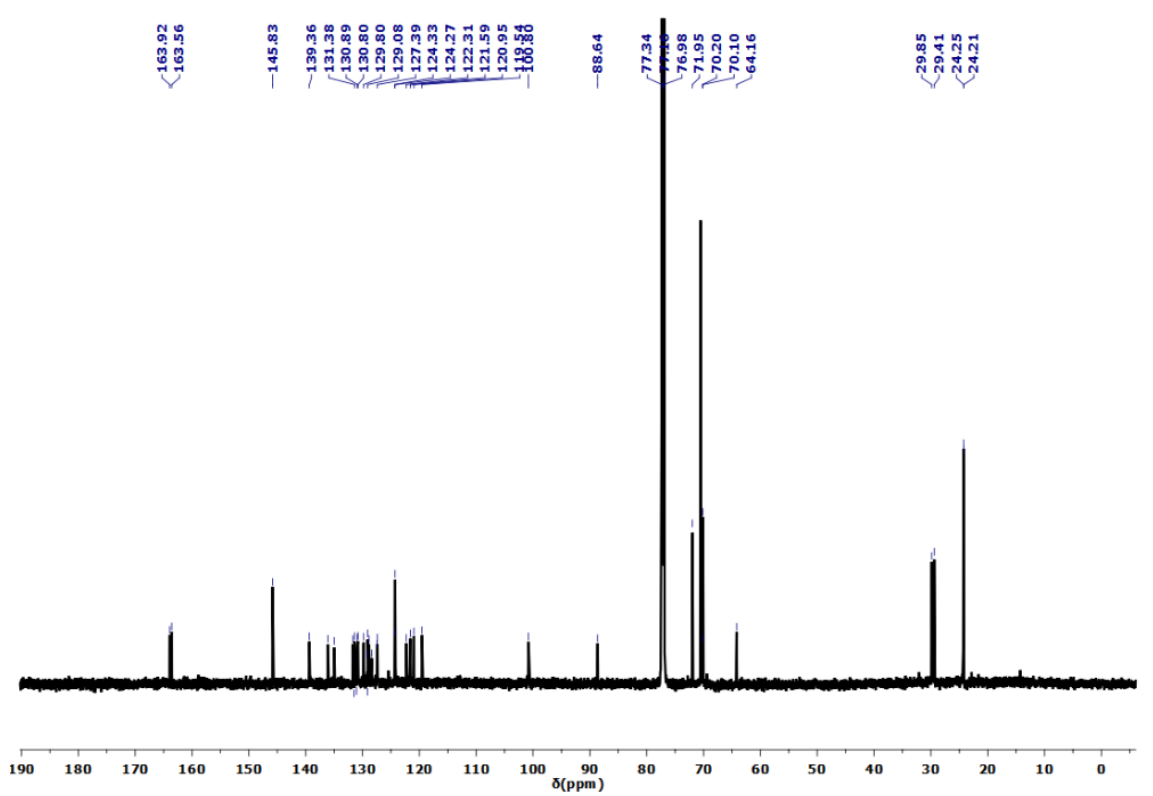

Figure S18: ${ }^{13} \mathrm{C}\left\{{ }^{1} \mathrm{H}\right\} \mathrm{NMR}$ spectra of $\mathbf{5 b}\left(175 \mathrm{MHz}, \mathrm{CDCl}_{3}, 298 \mathrm{~K}\right)$ 
a)

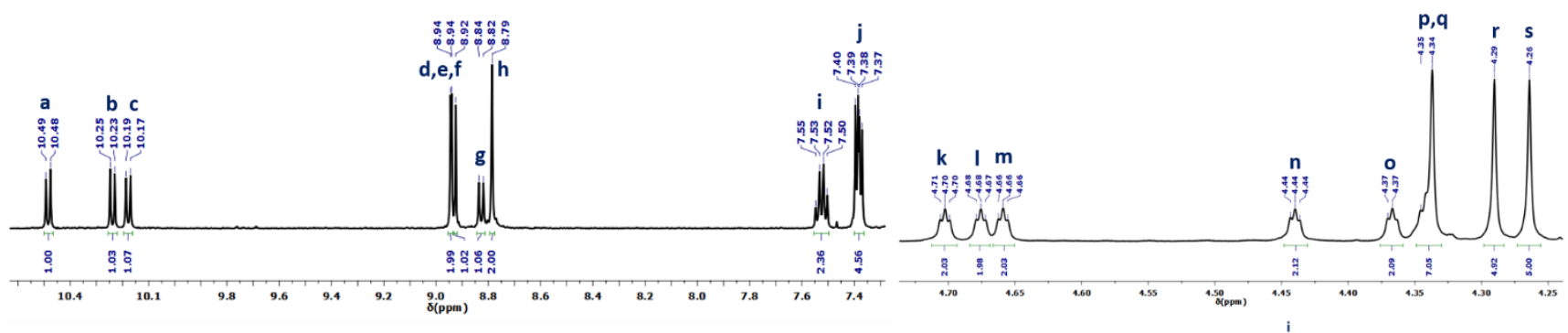

b)

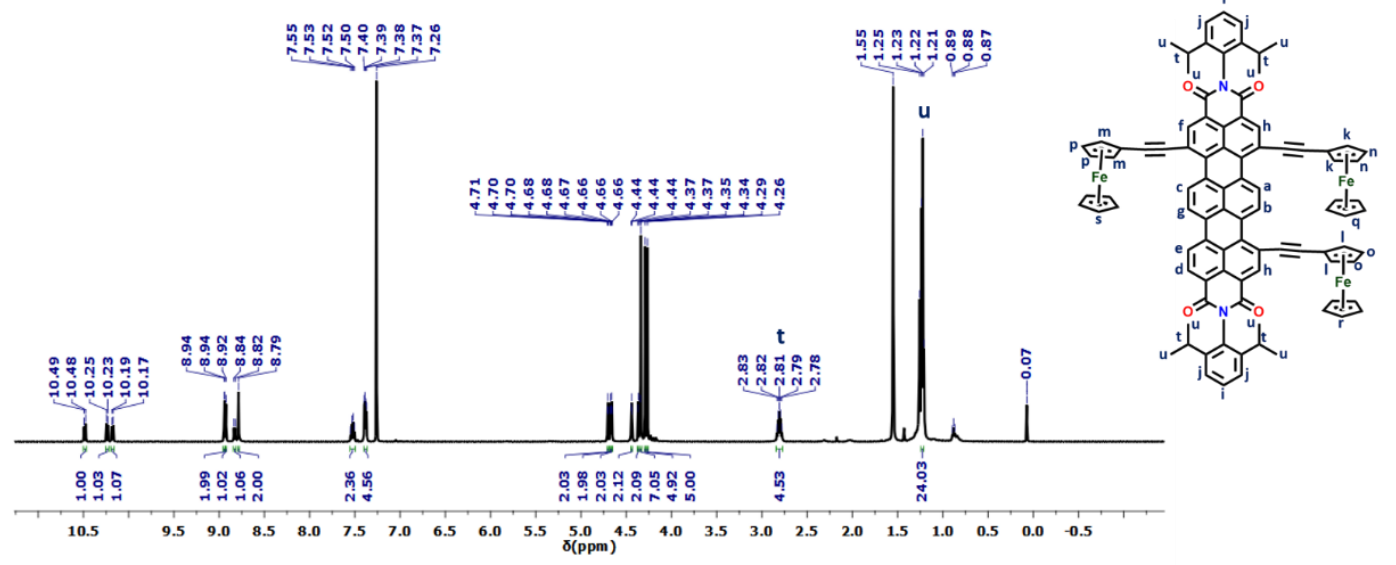

Figure S19: a) zoomed-in and b) full ${ }^{1} \mathrm{H}$ NMR spectra of 6 (500 MHz, $\mathrm{CDCl}_{3}, 298 \mathrm{~K}$ )

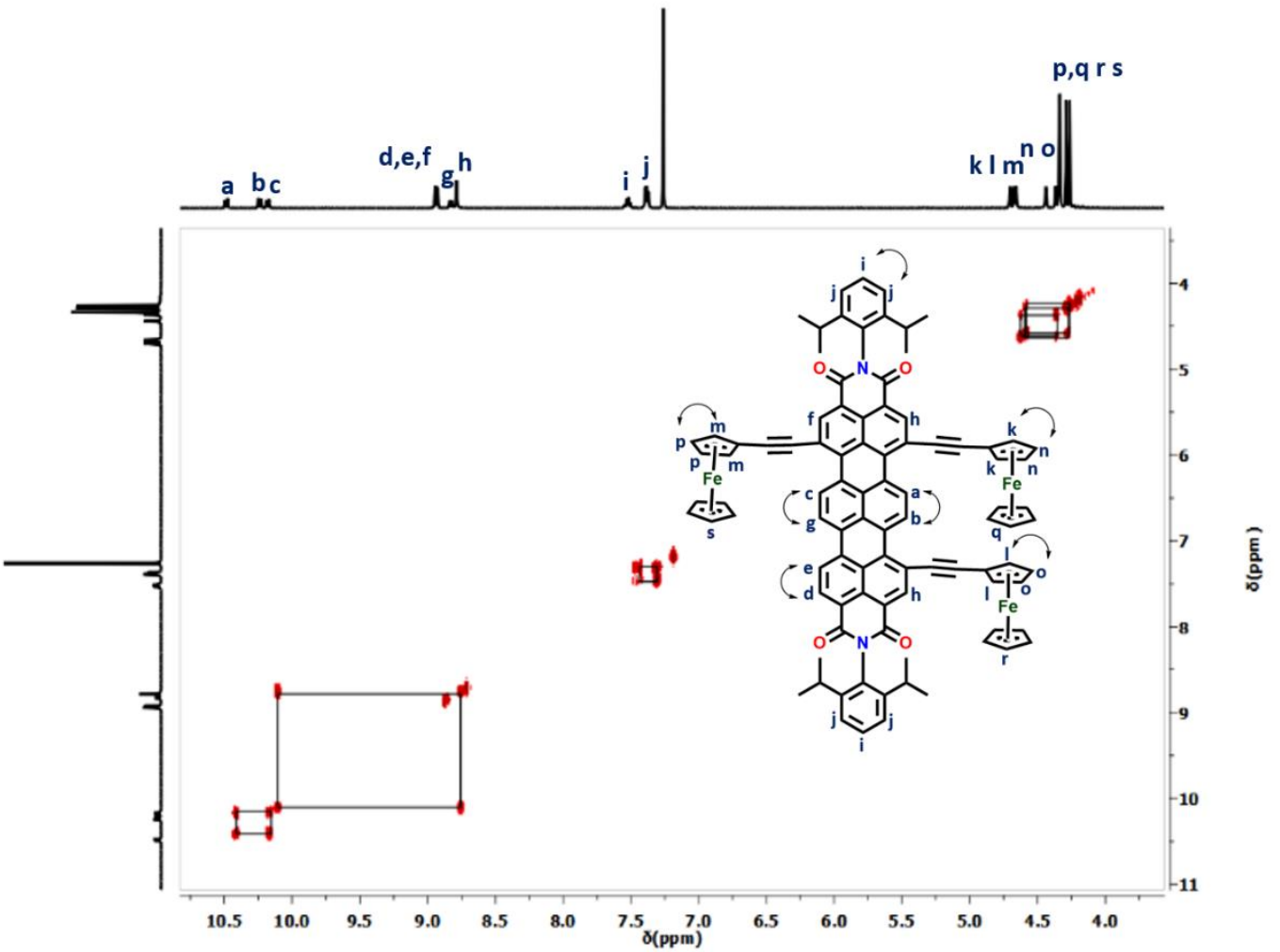

Figure S20: ${ }^{1} \mathrm{H}-1{ }^{1} \mathrm{H}$ Correlation (COSY) NMR of $6\left(500 \mathrm{MHz}, \mathrm{CDCl}_{3}, 298 \mathrm{~K}\right)$ 


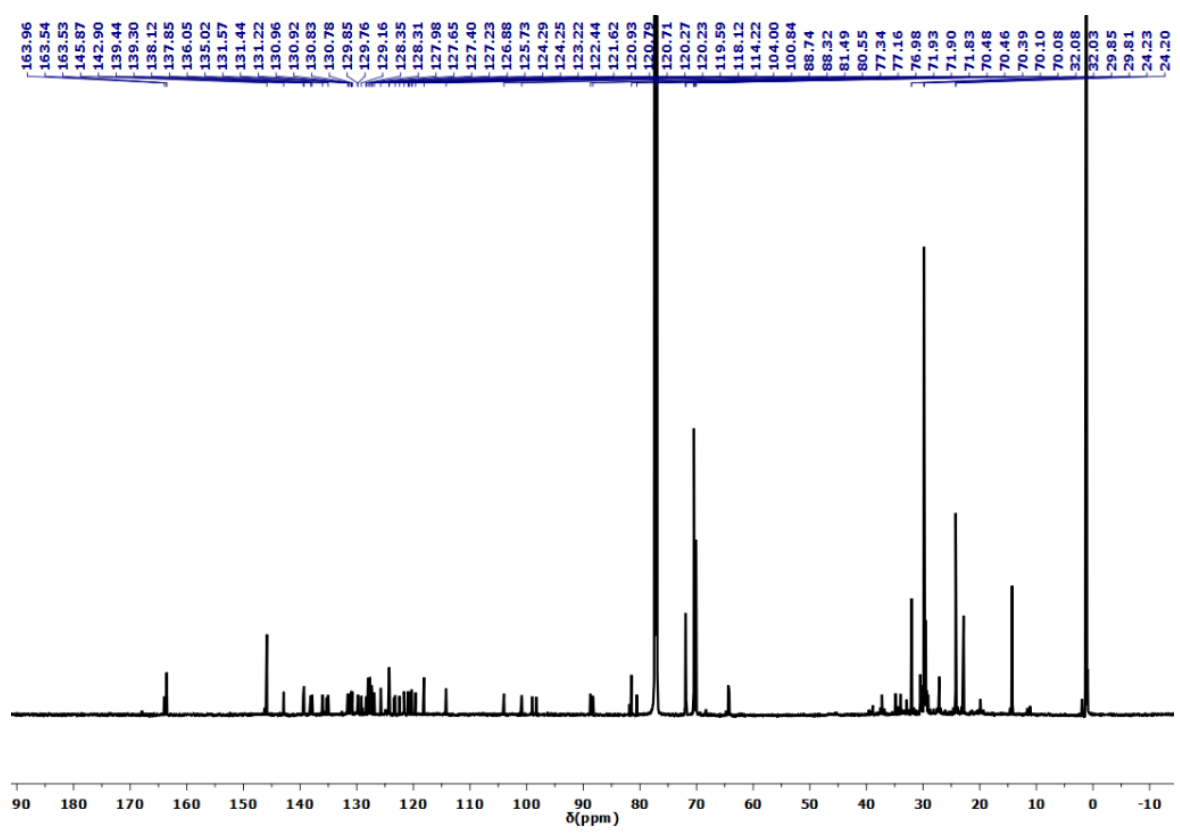

Figure S21: ${ }^{13} \mathrm{C}\left\{{ }^{1} \mathrm{H}\right\}$ NMR spectra of $6\left(175 \mathrm{MHz}, \mathrm{CDCl}_{3}, 298 \mathrm{~K}\right)$

\section{5: MASS SPECTRA}
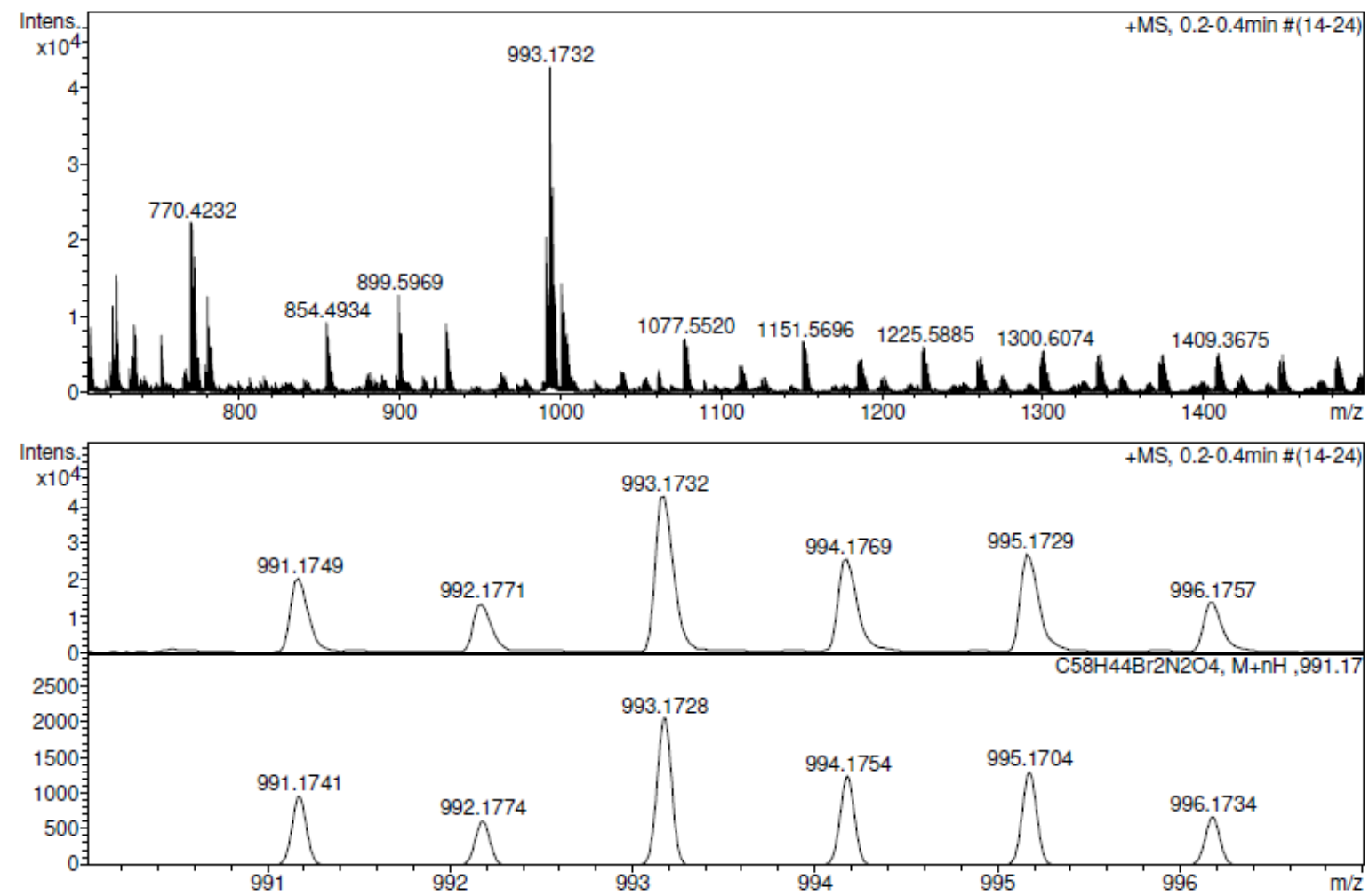

Figure S22: HRMS (APCI/Q-TOF) spectra of 2a 


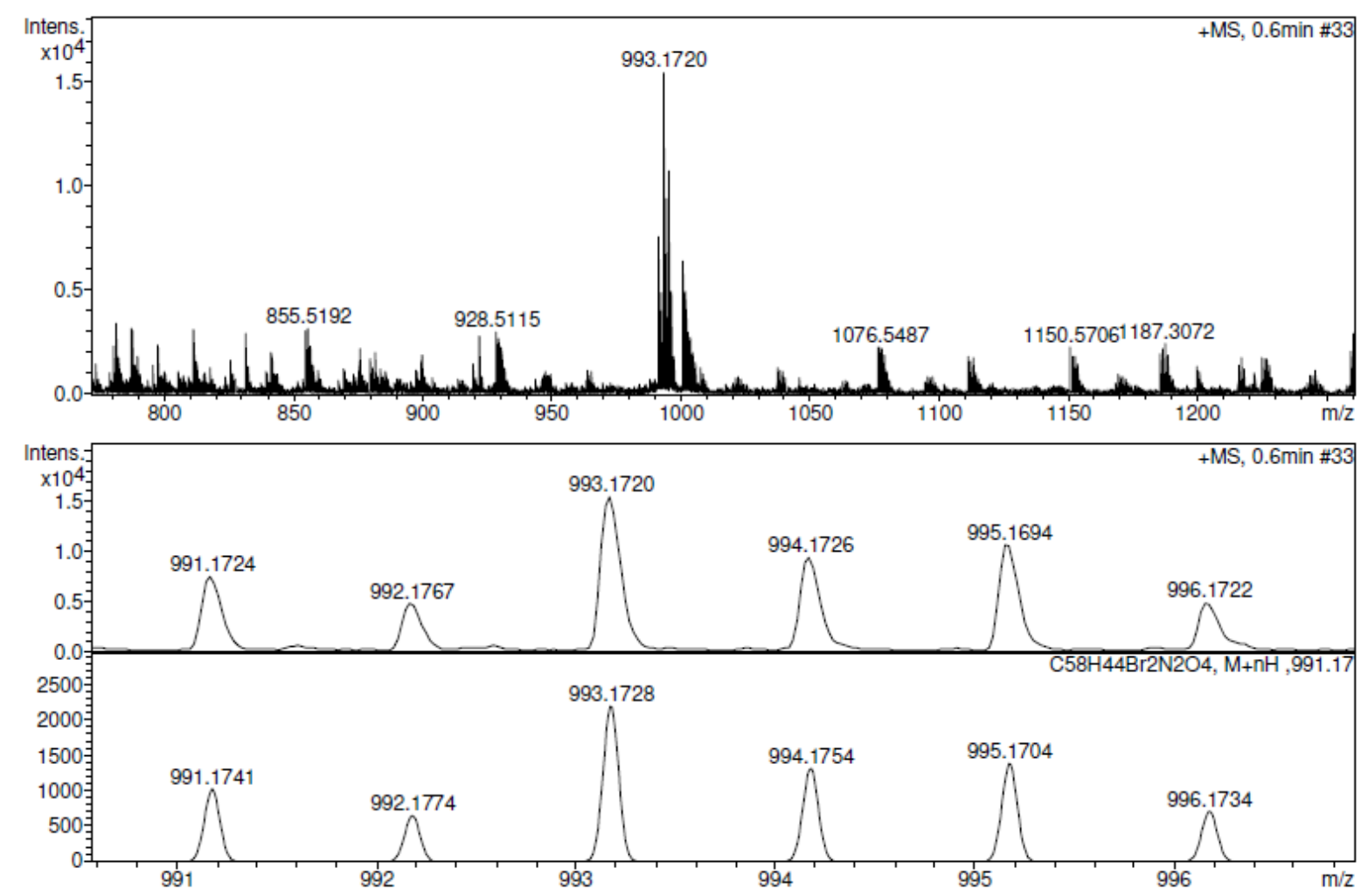

Figure S23: HRMS (APCI/Q-TOF) spectra of $2 \mathbf{b}$
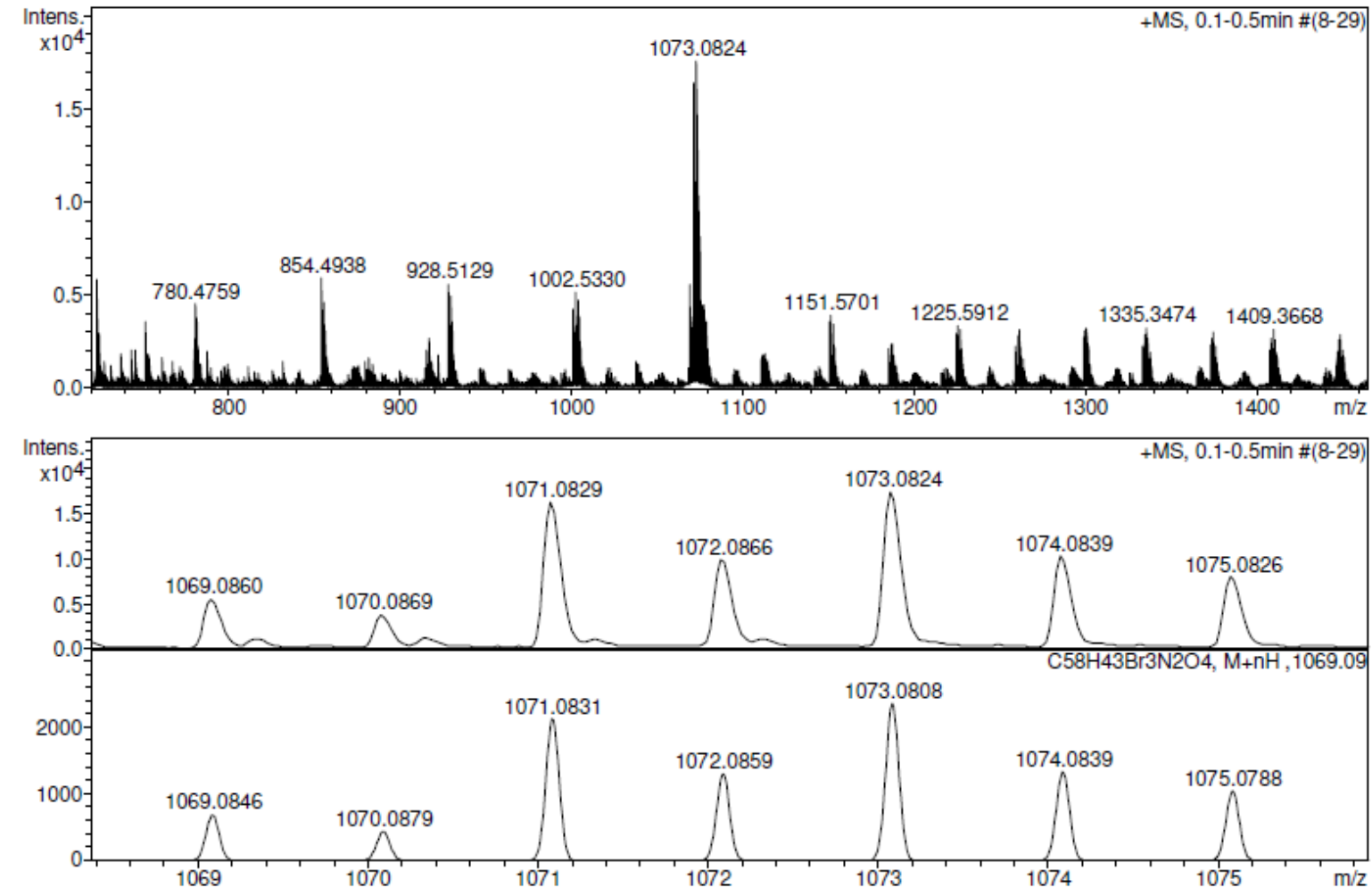

Figure S24: HRMS (APCI/Q-TOF) spectra of 3 

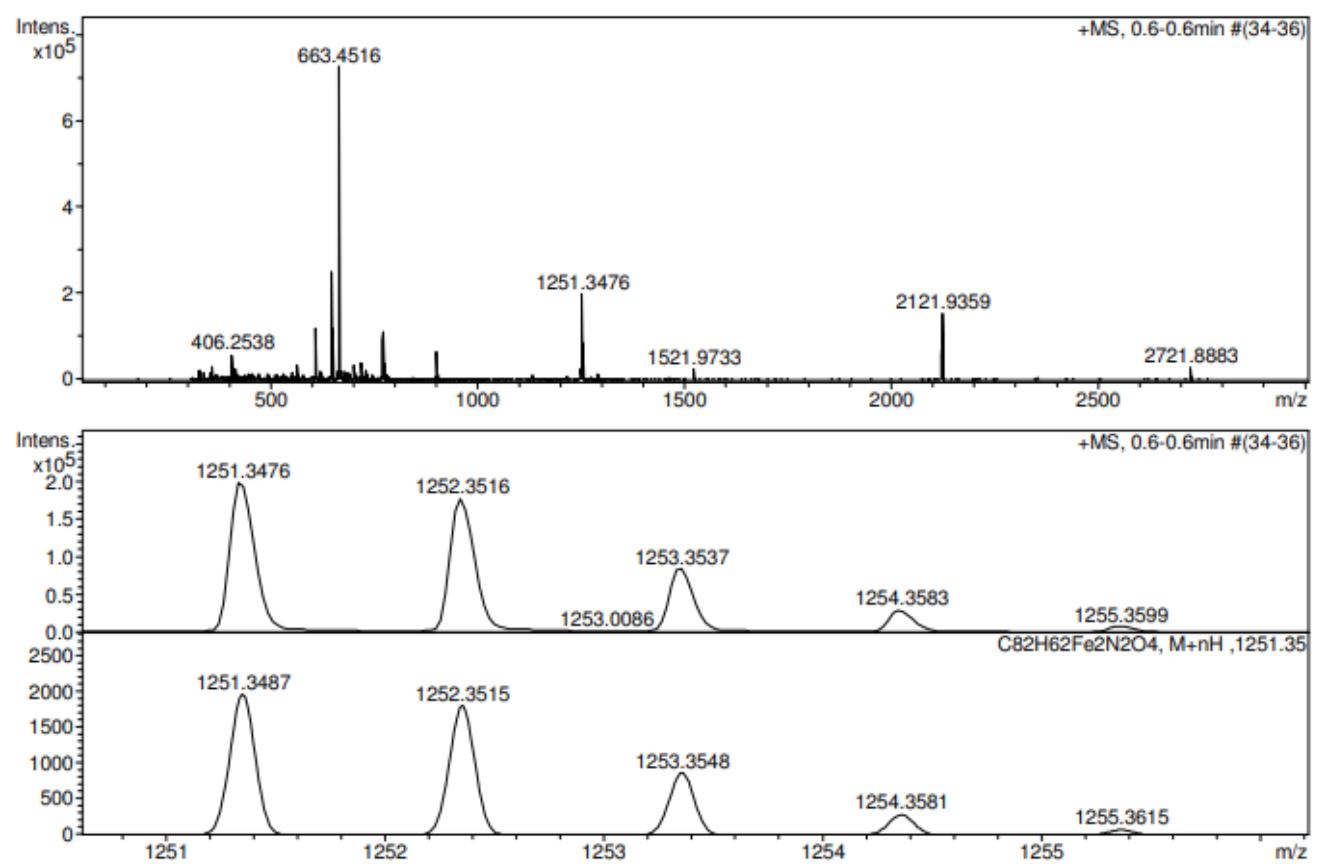

Figure S25: HRMS (APCI/Q-TOF) spectra of 5a

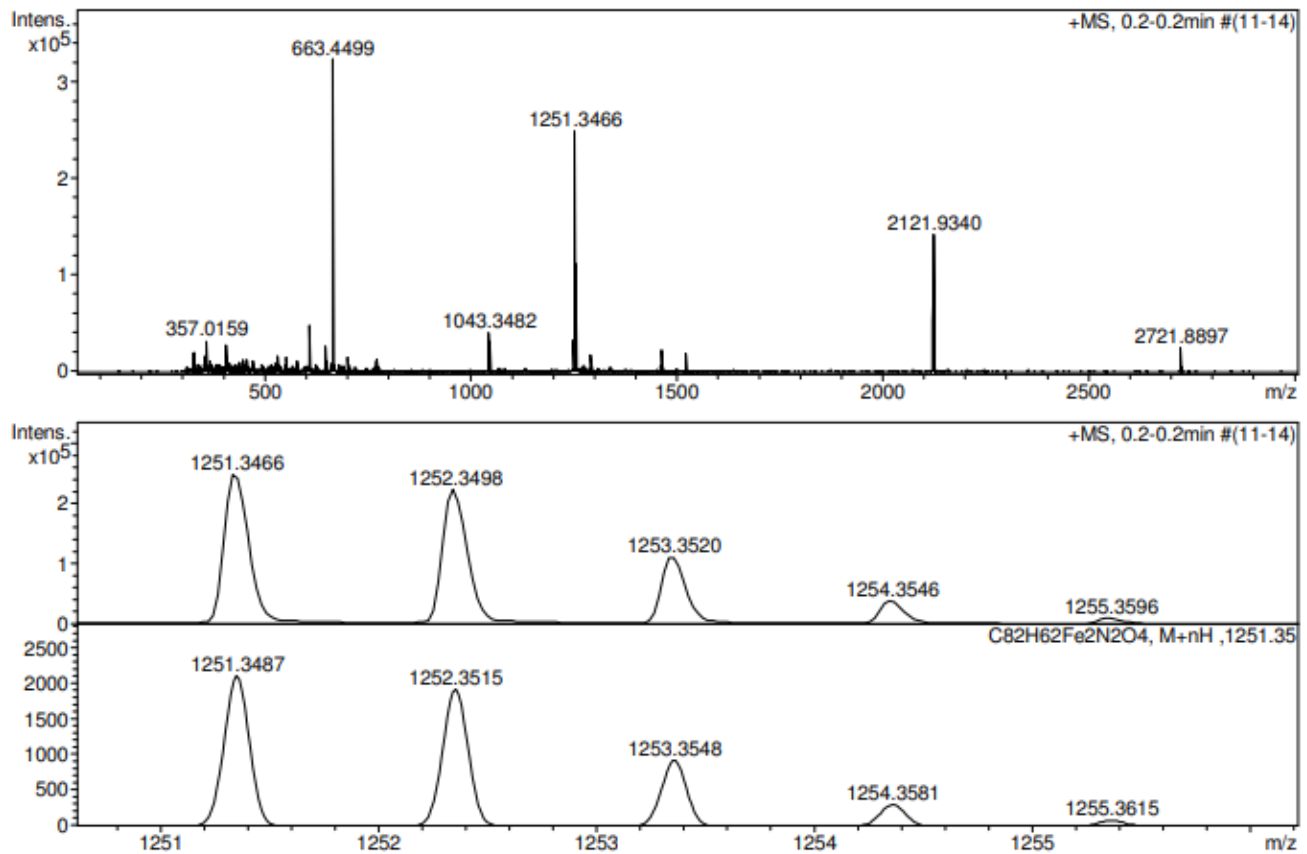

Figure S26: HRMS (APCI/Q-TOF) spectra of $\mathbf{5 b}$ 

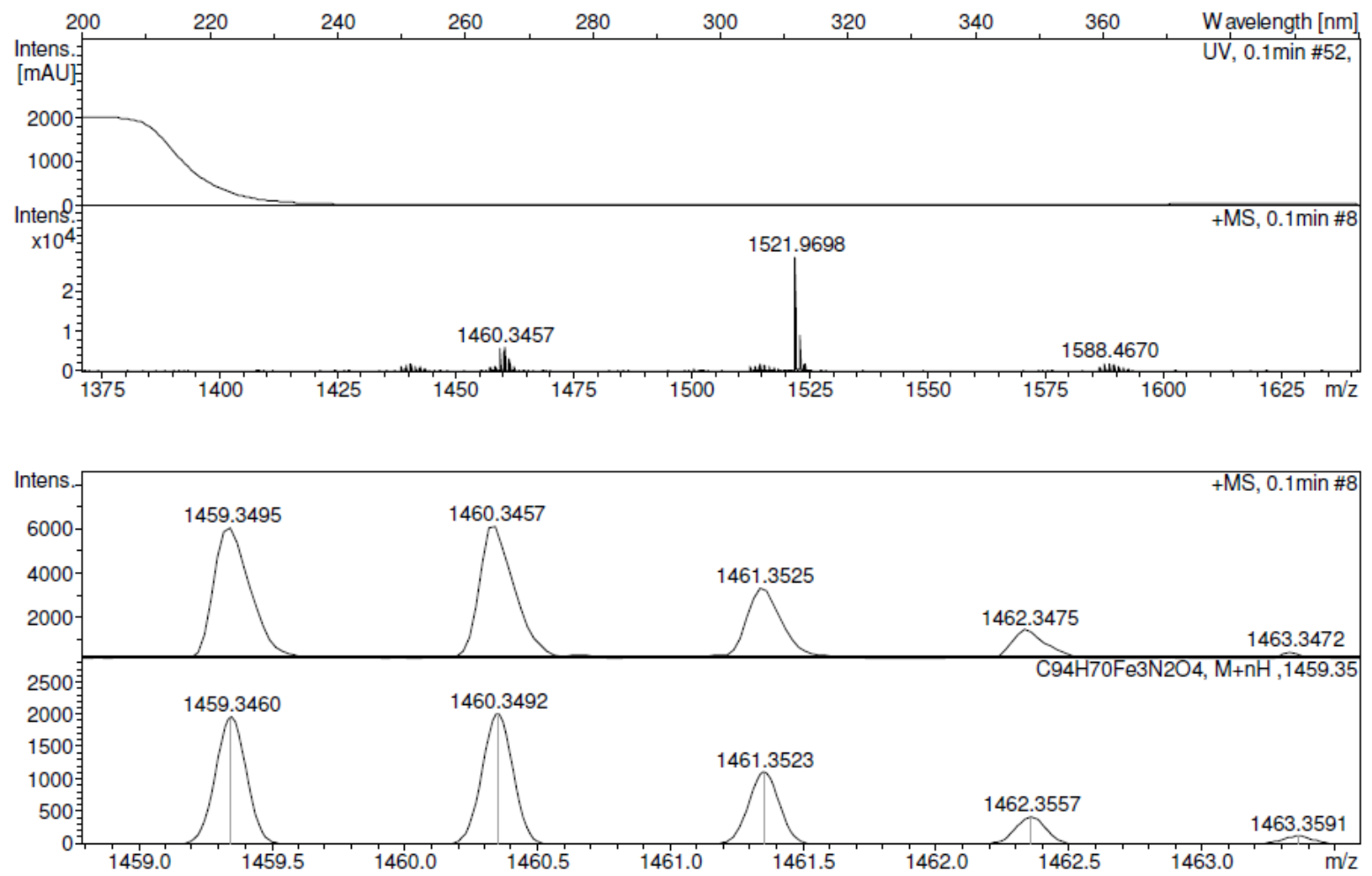

Figure S27: HRMS (APCI/Q-TOF) spectra of 6

6: Photo-physical studies

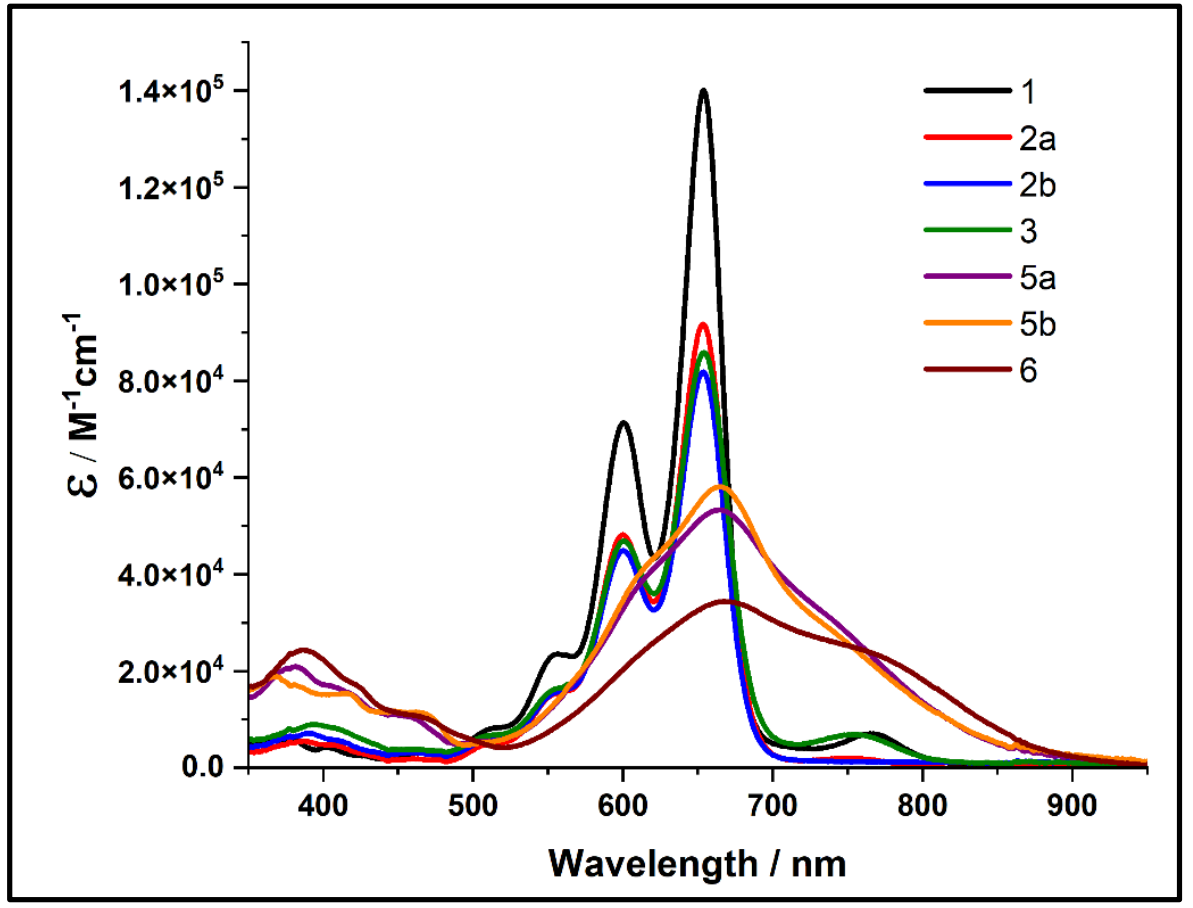

Figure S28: UV-vis-NIR Absorption Spectra of compounds 1, 2a, 2b, 3, 5a, 5b, and 6 in $\mathrm{CHCl}_{3}\left(2 \times 10^{-6} \mathrm{M}\right)$. 


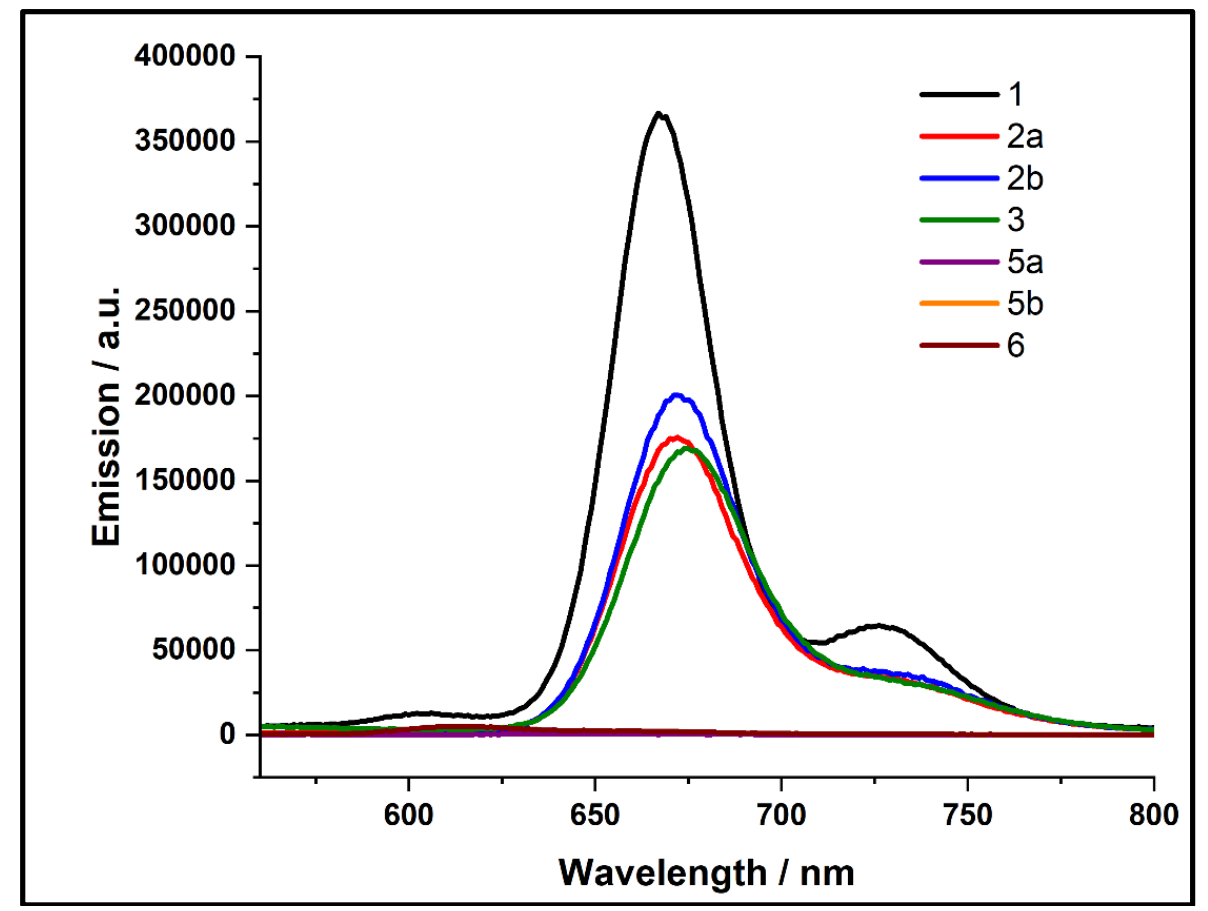

Figure S29 Fluorescence Emission Spectra of compounds 1, 2a, 2b, 3, 5a, 5b, and $\mathbf{6}$ in $\mathrm{CHCl}_{3}\left(2 \times 10^{-6} \mathrm{M}\right)$ (Excitation $550 \mathrm{~nm}$ )

\section{7: Electrochemical Studies}

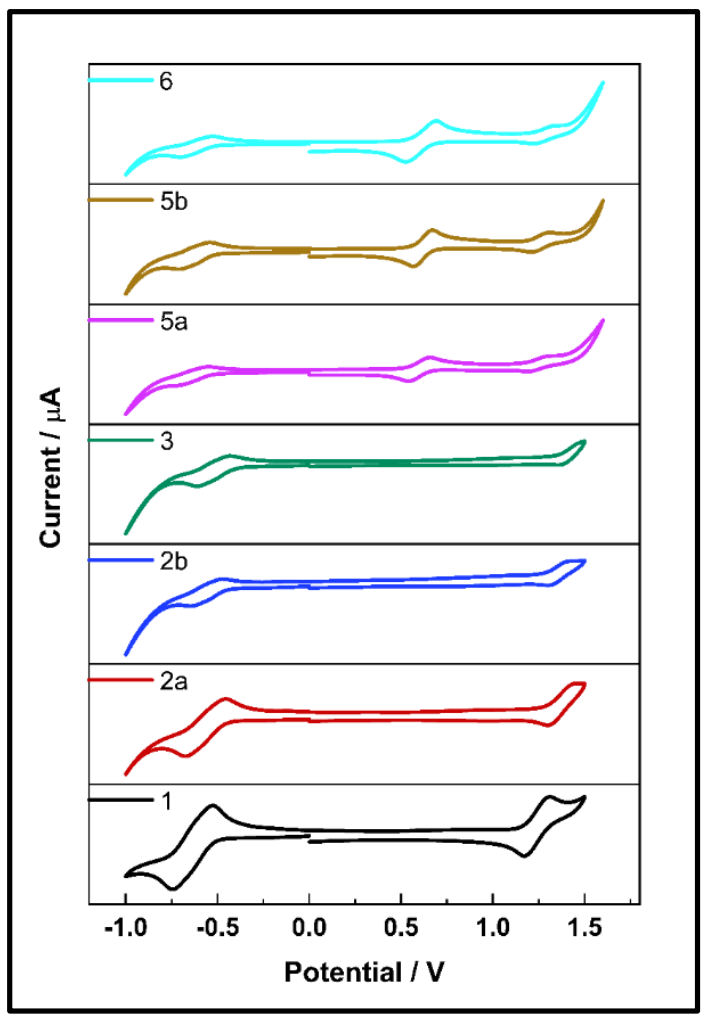

Figure S30 Cyclic voltammograms at scan rate: $0.05 \mathrm{~V} / \mathrm{s}$ of $\mathbf{1}, \mathbf{2 a}, \mathbf{2 b}, \mathbf{3}, \mathbf{5 a}, \mathbf{5 b}$, and $\mathbf{6}$ in chloroform containing $0.1 \mathrm{M}$ TBAPF as supporting electrolyte 
a)

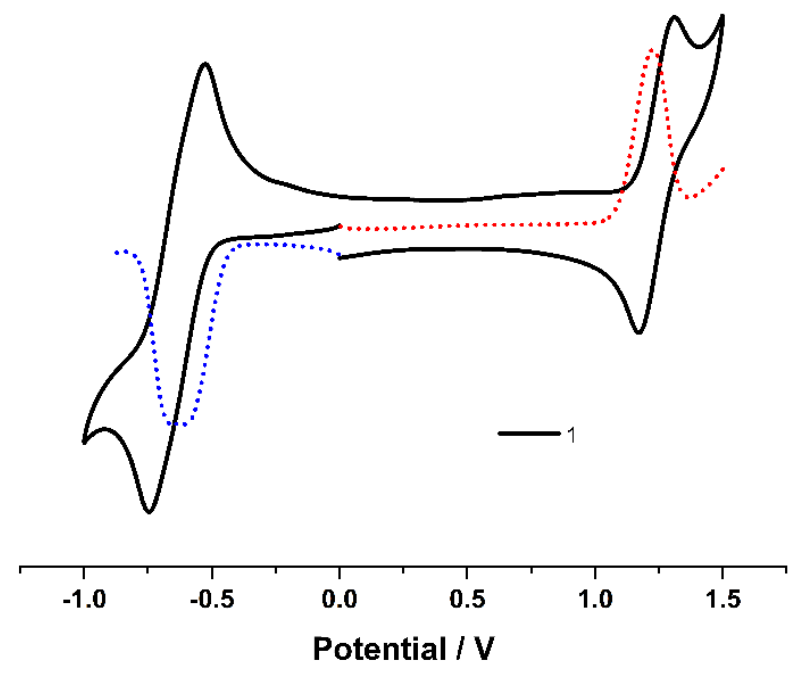

c)
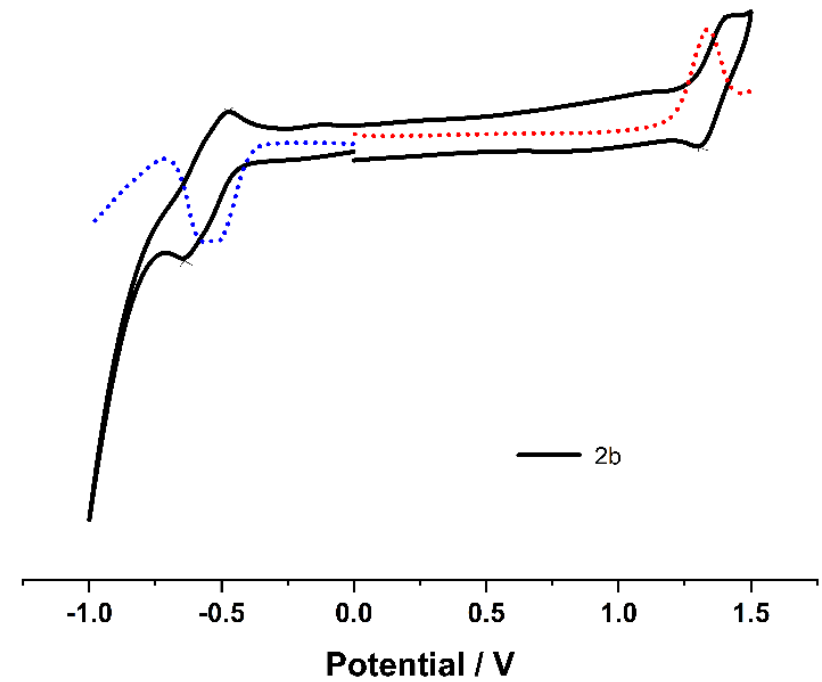

b)
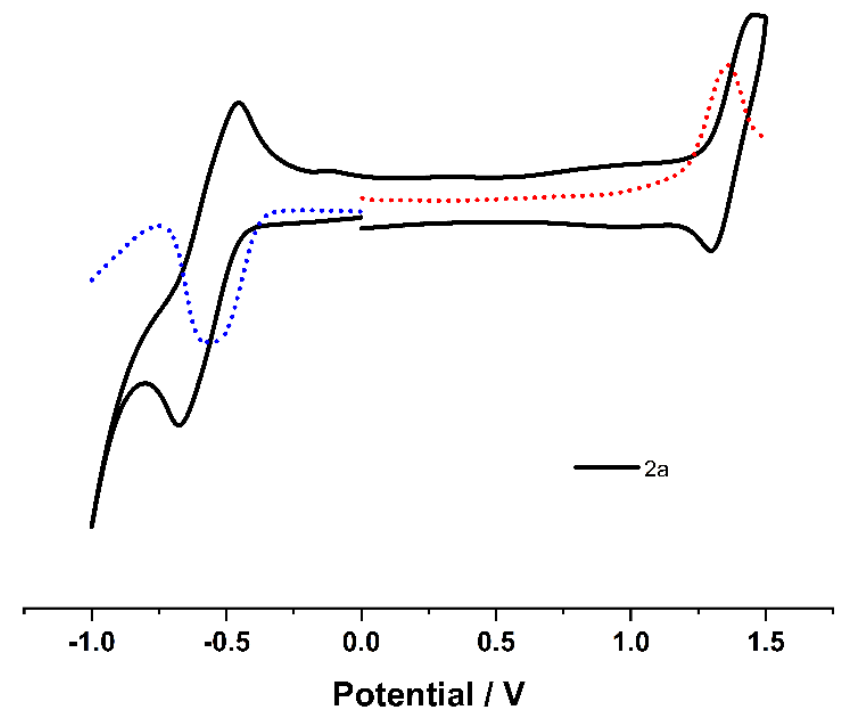

d)
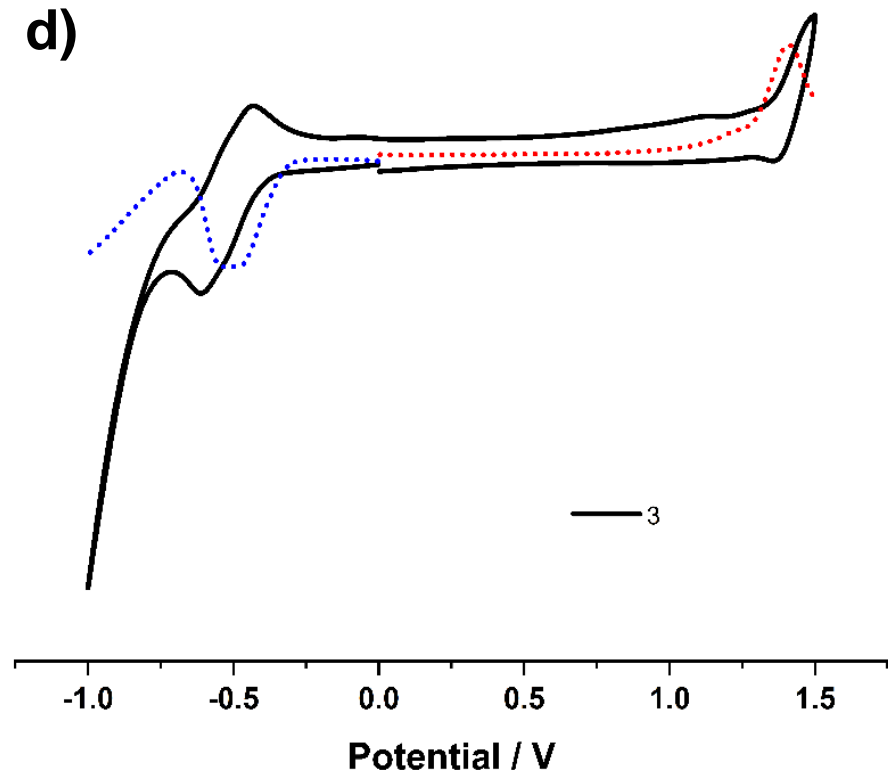
e)

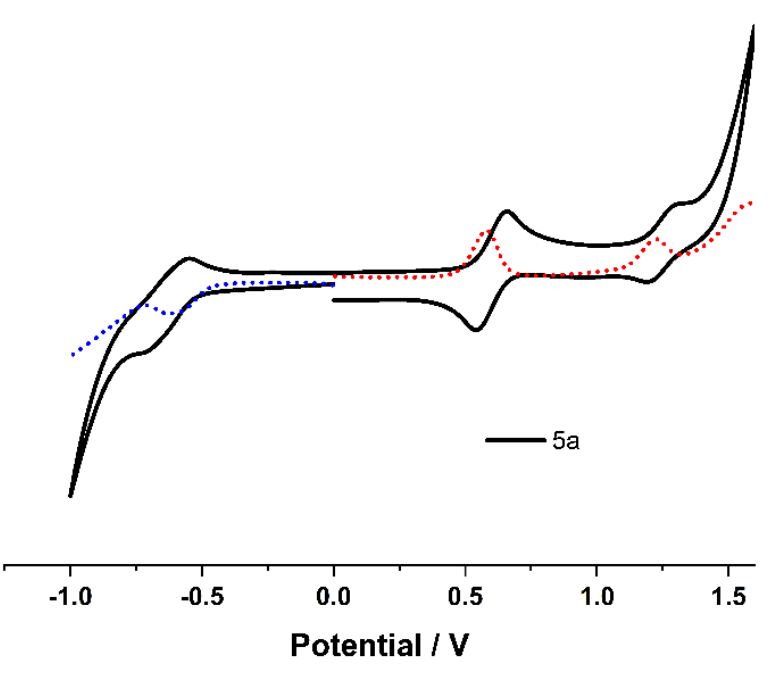

f)

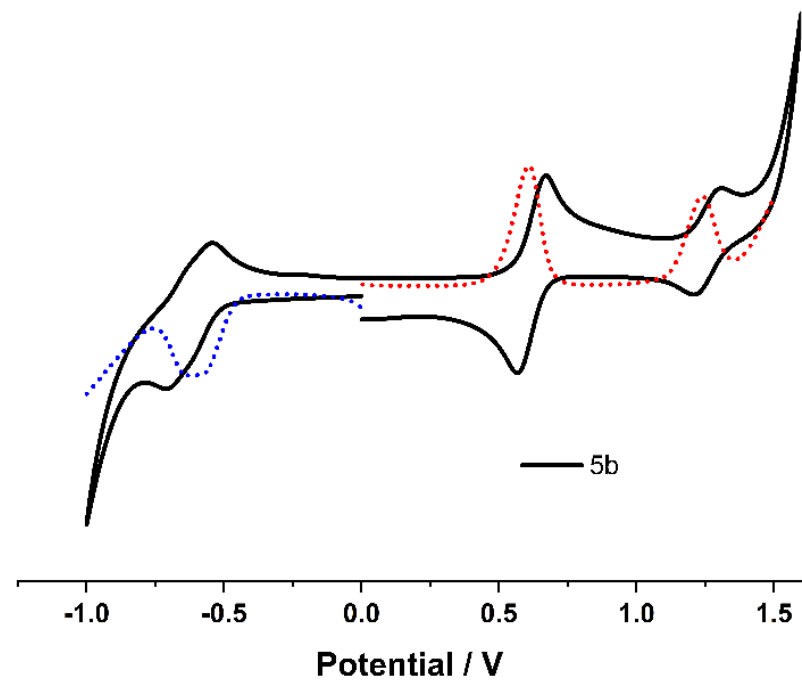

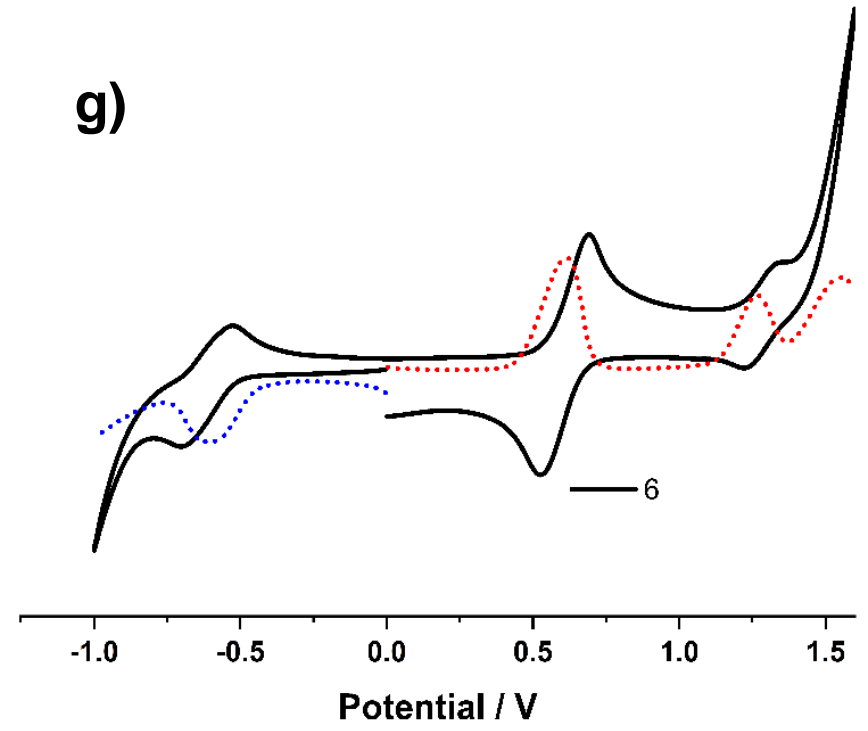

Figure S31 Bold lines and dotted lines showing cyclic and differential pulse voltammetry (a-g) respectively, for 1, 2a, $\mathbf{2 b}, \mathbf{3}, \mathbf{5 a}, \mathbf{5 b}$, and $\mathbf{6}$ in chloroform containing 0.1 M TBAPF as supporting electrolyte 


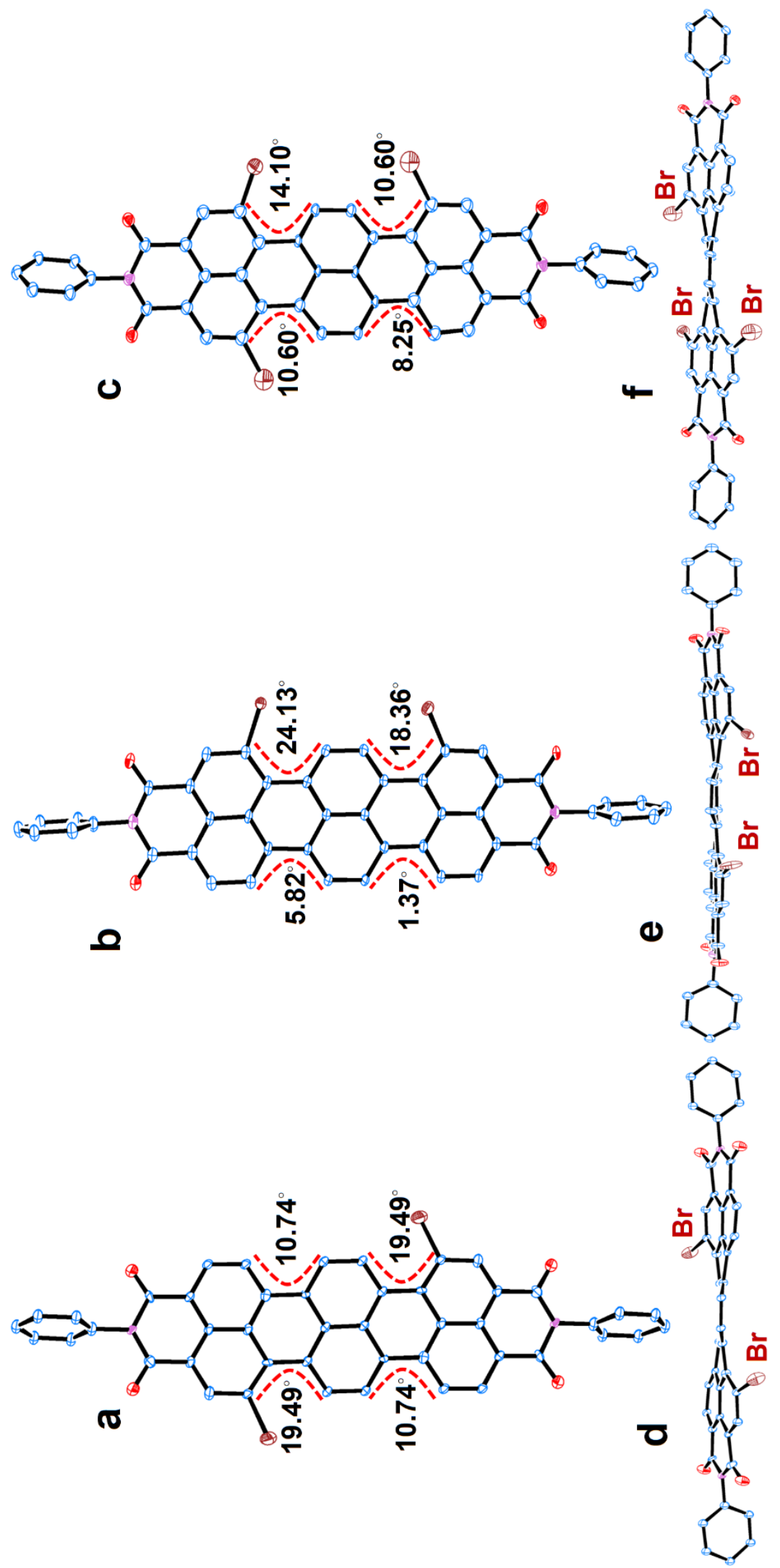

Figure S32 ORTEPs depicting the solid-state structures and torsion angles for 2a, 2b and $\mathbf{3}$ (CCDC 2121764, 2121765, 2121766) respectively. Top views(a,b,c) and side views(d,e,f). 